\title{
Decomposing finitely generated groups into free products with amalgamation
}

\author{
V. V. Benyash-Krivets
}

\begin{abstract}
The problem of the existence of a decomposition of a finitely generated group $\Gamma$ into a non-trivial free product with amalgamation is studied. It is proved that if $\operatorname{dim} X^{s}(\Gamma) \geqslant 2$, where $X^{s}(\Gamma)$ is the character variety of irreducible representations of $\Gamma$ into $\mathrm{SL}_{2}(\mathbb{C})$, then $\Gamma$ is a non-trivial free product with amalgamation. Next, the case when $\Gamma=\left\langle a, b \mid a^{n}=b^{k}=R^{m}(a, b)\right\rangle$ is a generalized triangle group is considered. It is proved that if one of the generators of $\Gamma$ has infinite order, then $\Gamma$ is a non-trivial free product with amalgamation. In the general case sufficient conditions ensuring that $\Gamma$ is a non-trivial free product with amalgamation are found.

Bibliography: 26 titles.
\end{abstract}

\section{Introduction}

We shall say that a group $G$ is a non-trivial free product with amalgamation if $G=G_{1} *_{A} G_{2}$, where $G_{1} \neq A \neq G_{2}$ (see [1]). Wall [2] posed the following question:

What one-relator groups are non-trivial free products with amalgamation?

Let $G=\left\langle g_{1}, \ldots, g_{m} \mid R_{1}=\cdots=R_{n}=1\right\rangle$ be a group with $m$ generators and $n$ relations such that $\operatorname{def} G=m-n \geqslant 2$. It is proved in [3] that $G$ is a non-trivial free product with amalgamation. In particular, if $G$ is a one-relator group with $m$ generators, $m \geqslant 3$, then $G$ is a non-trivial free product with amalgamation. The case of groups with two generators and one relation is more complicated. For example, the free Abelian group $G=\langle a, b \mid[a, b]=1\rangle$ of rank 2, where $[a, b]=$ $a b a^{-1} b^{-1}$, is obviously not a non-trivial free product with amalgamation. Another example is the group $G_{n}=\left\langle a, b \mid a b a^{-1}=b^{n}\right\rangle$. This group is soluble for each $n$, and bearing in mind results of [3] it is easy to show that $G_{n}$ is not a non-trivial free product with amalgamation for $n \neq-1$.

The following conjecture was stated in [4].

Conjecture 1. Let $G=\left\langle a, b \mid R^{m}(a, b)=1\right\rangle, m \geqslant 2$, be a group with two generators and one relation with torsion. Then $G$ is a non-trivial free product with amalgamation.

This research was carried out with the financial support of the Institute of Mathematics of the National Academy of Sciences in the framework of the State Basic Research Program "Mathematical Structures".

AMS 2000 Mathematics Subject Classification. Primary 20E06; Secondary 20F05. 
Zieschang [5] has studied the problem of the decomposition of discontinuous transformation groups into non-trivial free products with amalgamation. He has completely answered the question of when such a group is a non-trivial free product with amalgamation in all cases except for the groups $H_{1}=\left\langle a, b \mid[a, b]^{n}=1\right\rangle$ and $H_{2}=\left\langle a, b \mid a^{2}=[a, b]^{n}=1\right\rangle, n \geqslant 2$. Rosenberger [6] has proved that $H_{1}$ and $H_{2}$ are non-trivial free products with amalgamation if $n$ is not a power of 2 . It is shown in the recent papers [7] and [8] that $H_{1}$ is a non-trivial free product with amalgamation for arbitrary $n \geqslant 2$. An independent proof of this fact was given in [9], [10].

In the present paper we study a more general case; namely, we consider so-called generalized triangle groups $G$ having a presentation of the following form:

$$
G=\left\langle a, b \mid a^{m}=b^{n}=R^{l}(a, b)=1\right\rangle,
$$

where $l \geqslant 2$ and $R(a, b)$ is a cyclically reduced word in the free group on $a$ and $b$. Not all these groups are non-trivial free products with amalgamation. For example, Zieschang [5] has proved that the ordinary triangle group

$$
T(m, n, l)=\left\langle a, b \mid a^{m}=b^{n}=(a b)^{l}=1\right\rangle,
$$

where $m, n, l \geqslant 2$, is not a non-trivial free product with amalgamation. On the other hand, it is shown in [10] that every group $G$ with a presentation $\langle a, b| a^{2 m}=$ $\left.R^{l}(a, b)=1\right\rangle$, where $m \geqslant 0$ and $l \geqslant 2$, is a free product with amalgamation. Theorems 2 and 3 of the present paper contain more general results about the decomposition of generalized triangle groups into non-trivial free products with amalgamation.

We prove in Theorem 1 that a finitely generated group $\Gamma$ is a non-trivial free product with amalgamation if the dimension of some algebraic variety (the socalled character variety of irreducible representations of $\Gamma$ into $\mathrm{SL}_{2}(\mathbb{C})$ ) is larger than 1. To formulate this result we recall notation and some facts from geometric representation theory (see also [11]-[14]).

Let $\Gamma=\left\langle g_{1}, \ldots, g_{m}\right\rangle$ be a finitely generated group and let $G \subset \mathrm{GL}_{n}(K)$ be a connected linear algebraic group defined over an algebraically closed field $K$ of characteristic zero. Obviously, for each homomorphism $\rho: \Gamma \rightarrow G(K)$ the set of elements

$$
\left(\rho\left(g_{1}\right), \ldots, \rho\left(g_{m}\right)\right) \in G(K)^{m}=G(K) \times \cdots \times G(K)
$$

satisfies all defining relations of $\Gamma$. Hence the correspondence $\rho \rightarrow\left(\rho\left(g_{1}\right), \ldots, \rho\left(g_{m}\right)\right)$ is a bijection between $\operatorname{Hom}(\Gamma, G(K))$ and the set of $K$-points in some affine $K$ variety $R(\Gamma, G)$ in $G^{m}$. The variety $R(\Gamma, G)$ is usually called the representation variety of $\Gamma$ into the algebraic group $G$.

The group $G$ acts on $R(\Gamma, G)$ in the natural way (by simultaneous conjugation of components), and its orbits are in one-to-one correspondence with the equivalence classes of representations of $\Gamma$. In the general case the orbits of $G$ under this action are not necessarily closed and therefore the variety of orbits (the geometric quotient) is not an algebraic variety. However, if $G$ is a reductive group, then one can consider the categorical quotient $X(\Gamma, G)=R(\Gamma, G) / G$ (see [15]). Its points parametrize closed $G$-orbits. In the case when $G=\mathrm{GL}_{n}(K)$ or $G=\mathrm{SL}_{n}(K)$, an orbit of $G$ 
is closed if and only if the corresponding representation in completely reducible. Hence points in the variety $X(\Gamma, G)$ are in this case in one-to-one correspondence with the equivalence classes of completely reducible representations of $\Gamma$ into $G$ or, in other words, with the characters of representations of $\Gamma$ into $G$.

Throughout the paper we shall consider only the case $G=\mathrm{SL}_{2}(K)$ and for brevity set $R\left(\Gamma, \mathrm{SL}_{2}(K)\right)=R(\Gamma)$ and $X\left(\Gamma, \mathrm{SL}_{2}(K)\right)=X(\Gamma)$. One can find all information about the varieties $R(\Gamma)$ and $X(\Gamma)$ used below in [12] and [16]-[18]. We set

$$
R^{s}(\Gamma)=\{\rho \in R(\Gamma): \rho \text { is irreducible }\}, \quad X^{s}(\Gamma)=\pi\left(R^{s}(\Gamma)\right),
$$

where $\pi: R(\Gamma) \rightarrow X(\Gamma)$ is the canonical projection. It is shown in [12] that $R^{s}(\Gamma)$ and $X^{s}(\Gamma)$ are Zariski open subsets of $R(\Gamma)$ and $X(\Gamma)$ respectively.

The aim of the present paper is to prove the following results.

Theorem 1. Let $\Gamma$ be a finitely generated group such that $\operatorname{dim} X^{s}(\Gamma) \geqslant 2$. Then $\Gamma$ is a non-trivial free product with amalgamation.

Theorem 2. Suppose that $\Gamma_{n}=\left\langle a, b \mid a^{n}=b^{k}=R^{m}(a, b)=1\right\rangle$, where $n, k, m \in \mathbb{Z}, n, k, m \geqslant 2$, and $R(a, b)=a^{u_{1}} b^{v_{1}} \cdots a^{u_{s}} b^{v_{s}}$ is a word such that $0<u_{i}<n, 0<v_{i}<k$, and $s \geqslant 1$. Suppose that there exists $i \in\{1, \ldots, s\}$ such that $\left|u_{i}\right| \geqslant 2$. Moreover, suppose that $n=u_{i} p f$, where $f \in \mathbb{Z}, p$ is a prime, and $u_{i} p$ does not divide $u_{j}$ for $j \neq i$. Then $\Gamma_{n}$ is a non-trivial free product with amalgamation in the following cases:

(1) $m=2$ and $p$ does not belong to a certain finite set of primes $S$. The set $S$ is completely determined by the exponent $k$ and the word $R$.

(2) $m=3$ or $m=2^{l}>3, p \neq 2$.

(3) $m>3$ and $m \neq 2^{l}$.

Note that the condition $u_{i} p \nmid u_{j}$ for $j \neq i$ in Theorem 2 holds automatically if $u_{i}=\max _{1 \leqslant j \leqslant s} u_{j} \geqslant 2$ or $u_{i} \nmid u_{j}$ for each $j \neq i$.

Theorem 3. Suppose that $\Gamma=\left\langle a, b \mid a^{n}=R^{m}(a, b)=1\right\rangle$, where $n=0$ or $n \geqslant 2$, $m \geqslant 2$, and $R(a, b)=a^{u_{1}} b^{v_{1}} \cdots a^{u_{s}} b^{v_{s}}$ with $s \geqslant 1, v_{i} \neq 0$, and $0<u_{i}<n$. Then $\Gamma$ is a non-trivial free product with amalgamation.

As an immediate consequence of Theorem 3 we obtain the proof of Conjecture 1.

Corollary 1. Let $\Gamma=\left\langle a, b \mid R^{m}(a, b)=1\right\rangle, m \geqslant 2$, be a group with two generators and one relation with torsion. Then $\Gamma$ is a non-trivial free product with amalgamation.

At the end of $\S 2$ we shall prove that for $m \geqslant 3$ the group $\Gamma$ in Corollary 1 satisfies the assumptions of Theorem 1 , that is, $\operatorname{dim} X^{s}(\Gamma) \geqslant 2$, and therefore we obtain another proof of Conjecture 1.

Corollary 2. The Fuchsian groups $H_{1}=\left\langle a, b \mid[a, b]^{n}=1\right\rangle$ and $H_{2}=$ $\left\langle a, b \mid a^{2}=[a, b]^{n}=1\right\rangle, n \geqslant 2$, are non-trivial free products with amalgamation.

\section{$\S 1$. Proof of Theorem 1}

In what follows we denote the field of $p$-adic numbers by $\mathbb{Q}_{p}$, the ring of $p$-adic integers by $\mathbb{Z}_{p}$, the group of $p$-adic units in $\mathbb{Z}_{p}$ by $\mathbb{Z}_{p}^{*}$, the $p$-adic valuation by $|\cdot|_{p}$, the trace of a matrix $A$ by $\operatorname{tr} A$, and the identity $2 \times 2$-matrix by $E$. 
We recall several facts about the character variety $X(\Gamma)$ of representations of a finitely generated group $\Gamma$ into $\mathrm{SL}_{2}(\mathbb{C})$ (see $[12]$ ). For arbitrary $g$ in $\Gamma$ one can consider the regular function

$$
\tau_{g}: R(\Gamma) \rightarrow \mathbb{C}, \quad \tau_{g}(\rho)=\operatorname{tr} \rho(g) .
$$

Usually, $\tau_{g}$ is called the Fricke character of the element $g$. It is known that the $\mathbb{Z}$-algebra $T(\Gamma)$ generated by all functions $\tau_{g}, g \in \Gamma$, is finitely generated. Moreover, if $\tau_{g_{1}}, \ldots, \tau_{g_{s}}$ are generators of $T(\Gamma)$, then the $\mathbb{C}$-algebra of $\mathrm{SL}_{2}(\mathbb{C})$-invariant regular functions $\mathbb{C}[R(\Gamma)]^{\mathrm{SL}_{2}(\mathbb{C})}$ is equal to $\mathbb{C}\left[\tau_{g_{1}}, \ldots, \tau_{g_{s}}\right]$. Consider now the morphism

$$
\pi: R(\Gamma) \rightarrow \mathbb{A}^{s}, \quad \pi(\rho)=\left(\tau_{g_{1}}(\rho), \ldots, \tau_{g_{s}}(\rho)\right) .
$$

It is shown in [12] that the image $\pi(R(\Gamma))$ is closed in $\mathbb{A}^{s}$. Since $X(\Gamma)$ and $\pi(R(\Gamma))$ are biregularly isomorphic, we shall identify $X(\Gamma)$ and $\pi(R(\Gamma))$.

The idea of the proof of Theorem 1 is to construct for some prime $p$ a representation $\rho: \Gamma \rightarrow \mathrm{SL}_{2}\left(\mathbb{Q}_{p}\right)$ such that $\rho(\Gamma)$ is dense in $\mathrm{SL}_{2}\left(\mathbb{Q}_{p}\right)$ in the $p$-adic topology. After that the following well-known facts will yield Theorem 1 .

(1) If $H$ is a dense subgroup of $\mathrm{SL}_{2}\left(\mathbb{Q}_{p}\right)$ in the $p$-adic topology, then $H$ is a non-trivial free product with amalgamation (see [19]).

(2) If $f: G_{1} \rightarrow G_{2}$ is a group epimorphism and $G_{2}$ is a non-trivial free product with amalgamation, then $G_{1}$ is also a non-trivial free product with amalgamation.

We shall say that a subgroup $H$ of $\mathrm{SL}_{2}\left(\mathbb{Q}_{p}\right)$ is unbounded if $H$ does not lie in $\mathrm{SL}_{2}\left(\mathbb{Z}_{p}\left[p^{-s}\right]\right)$ for any $s \geqslant 1$.

Lemma 1. Let $H$ be a subgroup of $\mathrm{SL}_{2}\left(\mathbb{Q}_{p}\right)$. Then $H$ is dense in $\mathrm{SL}_{2}\left(\mathbb{Q}_{p}\right)$ in the p-adic topology if and only if $H$ is absolutely irreducible (that is, irreducible over the algebraic closure of $\mathbb{Q}_{p}$ ), unbounded, non-discrete, and does not lie in the normalizer of a maximal torus.

Proof. If $H$ is dense in $\mathrm{SL}_{2}\left(\mathbb{Q}_{p}\right)$ in the $p$-adic topology, then the assertion of the lemma is obvious. We now claim the converse result. Let $\bar{H}$ be the closure of $H$ in the $p$-adic topology. Then $\bar{H}$ is a $p$-adic Lie group. Let $\mathfrak{h}$ and $\mathfrak{s}$ be the Lie algebras of $\bar{H}$ and $\mathrm{SL}_{2}\left(\mathbb{Q}_{p}\right)$ respectively. We prove first that $\mathfrak{h}=\mathfrak{s}$. To this end it is sufficient to show by [7]; Theorem 4.6 that $\mathfrak{h}$ is not soluble. Assume the contrary, in which case $\bar{H}$ contains an open soluble subgroup $G$ (see [20]; Chapter 4). Let

$$
\Gamma_{j}=\left\{\left(\begin{array}{cc}
1+p^{j} a & p^{j} b \\
p^{j} c & 1+p^{j} d
\end{array}\right) \in \mathrm{SL}_{2}\left(\mathbb{Q}_{p}\right): a, b, c, d \in \mathbb{Z}_{p}\right\}, \quad j \geqslant 0,
$$

be the principal congruence subgroup of level $j$ of $\mathrm{SL}_{2}\left(\mathbb{Q}_{p}\right)$. The groups $\Gamma_{j}, j \geqslant 0$, form a base of neighbourhoods of the identity in $\mathrm{SL}_{2}\left(\mathbb{Q}_{p}\right)$. Hence we can assume without loss of generality that $G=\Gamma_{j} \cap \bar{H}$ for some $j \geqslant 2$. Since $\bar{H}$ it not discrete, $G$ is not discrete either. In particular, for each $i>j$ the group $G_{i}=\Gamma_{i} \cap \bar{H} \subset G$ is infinite.

We claim that $G$ is reducible over $\overline{\mathbb{Q}}_{p}$. For otherwise, in view of [21]; Corollary 2, $G$ contains a normal Abellian subgroup $A$ of index 2 and we have $\operatorname{tr} x=0$ for each $x$ in $G \backslash A$. On the other hand, if $x \in \mathrm{SL}_{2}\left(\mathbb{Q}_{p}\right)$ and $\operatorname{tr} x=0$, then $x \notin \Gamma_{i}$ for $i>1$; therefore $x \notin G$, which is a contradiction. 
To complete the proof of the insolubility of $\mathfrak{h}$, we consider the following cases.

(1) $G$ is Abelian. Since $H$ is absolutely irreducible and does not lie in the normalizer of a maximal torus, there exists $x \in H$ such that $x G x^{-1} \cap G=\{E\}$. Thus, we see that $\{E\}$ is an open subgroup of $G$, that is, $G$ is discrete, which is a contradiction.

(2) $G$ is non-Abelian. Then we can assume without loss of generality that all $G_{i}$ are non-Abelian for $i>j$ (otherwise we can set $G=G_{i}$ for some $i$ ). Hence the derived group $U=[G, G]$ is a non-trivial Abelian unipotent subgroup of $G$. By the absolute irreducibility of $H$ there exists $x$ in $H$ such that $x U x^{-1} \cap U=\{E\}$. On the other hand $x G x^{-1}$ is an open subgroup, and therefore there exists $i$ such that $G_{i} \subset x G x^{-1}$. Since $G_{i}$ is non-Abelian, it follows that $V=\left[G_{i}, G_{i}\right] \neq\{E\}$ and it is easy to see that $V \subset x U x^{-1} \cap U$, which is a contradiction.

We have thus proved that $\mathfrak{h}=\mathfrak{s}$. Hence there exists a congruence subgroup $\Gamma_{i}$ such that $\Gamma_{i} \subset \bar{H}$ (see [22]; Chapter. 5). In particular, $\bar{H}$ contains unipotent subgroups of the following form:

$$
U_{1}=\left\{\left(\begin{array}{cc}
1 & 0 \\
p^{i} a & 1
\end{array}\right): a \in \mathbb{Z}_{p}\right\}, \quad U_{2}=\left\{\left(\begin{array}{cc}
1 & p^{i} a \\
0 & 1
\end{array}\right): a \in \mathbb{Z}_{p}\right\} .
$$

Furthermore, the unboundedness of $H$ means that there exists an element $h$ of $H$ such that $|\operatorname{tr} h|_{p}>1$. For otherwise the traces of all elements of $H$ belong to $\mathbb{Z}_{p}$, and therefore $H$ is conjugate to a subgroup of $\mathrm{SL}_{2}\left(\mathbb{Z}_{p}\right)$ (see [23] or [12]; Lemma I.4.3), that is, $H$ is bounded. This is in contradiction with the assumptions of the theorem. We claim that the eigenvalues of the matrix $h$ belong to $\mathbb{Q}_{p}$. Suppose that $\operatorname{tr} h=$ $p^{-s} \alpha$, where $\alpha \in \mathbb{Z}_{p}^{*}, s>0$. Then the characteristic polynomial of $h$ has the form $f(y)=y^{2}-p^{-s} \alpha y+1$, and its discriminant is $D=p^{-2 s} \alpha^{2}-4=p^{-2 s}\left(\alpha^{2}-4 p^{2 s}\right)$. Thus, $D$ is a square in $\mathbb{Q}_{p}$, and therefore the roots of $f(y)$ belong to $\mathbb{Q}_{p}$. Hence $h$ is conjugate in $\mathrm{GL}_{2}\left(\mathbb{Q}_{p}\right)$ to a diagonal matrix of the following form:

$$
\operatorname{diag}\left(\lambda, \lambda^{-1}\right), \quad \lambda=p^{-s} \gamma, \quad s>0, \quad \gamma \in \mathbb{Z}_{p}^{*} .
$$

We can assume without loss of generality, taking into consideration a group conjugate to $H$ if necessary, that $h=\operatorname{diag}\left(\lambda, \lambda^{-1}\right) \in H$. It is now easy to show that $\bar{H}$ contains the following unipotent subgroups of $\mathrm{SL}_{2}\left(\mathbb{Q}_{p}\right)$ :

$$
V_{1}=\left\{\left(\begin{array}{ll}
1 & 0 \\
a & 1
\end{array}\right): a \in \mathbb{Q}_{p}\right\}, \quad V_{2}=\left\{\left(\begin{array}{ll}
1 & a \\
0 & 1
\end{array}\right): a \in \mathbb{Q}_{p}\right\}
$$

Indeed, let $x=\left(\begin{array}{cc}1 & 0 \\ p^{r} \beta & 1\end{array}\right)$ be an element of $V_{1}$, where $r<i$ and $\beta \in \mathbb{Z}_{p}^{*}$. We choose an integer $m$ such that $2 s m+r \geqslant i$. Then it is easy to see that $h^{m} x h^{-m} \in U_{1}$, and therefore $x \in \bar{H}$. Thus, we have $V_{1} \subset \bar{H}$, and in a similar way, $V_{2} \subset \bar{H}$. It is well-known that the subgroups $V_{1}$ and $V_{2}$ generate $\mathrm{SL}_{2}\left(\mathbb{Q}_{p}\right)$. Hence $\bar{H}=\mathrm{SL}_{2}\left(\mathbb{Q}_{p}\right)$, as required, and the proof is complete.

Lemma 2. Let $X$ and $Y$ be irreducible $\mathbb{Q}$-defined affine varieties, $\operatorname{dim} Y \geqslant 1$, and let $f: X \rightarrow Y$ be a dominant $\mathbb{Q}$-defined regular morphism. Then there exist a prime $p \neq 2$ and a point $x$ of $X\left(\mathbb{Q}_{p}\right)$ such that not all coordinates of the point $f(x)$ of $Y\left(\mathbb{Q}_{p}\right)$ belong to $\mathbb{Z}_{p}$. 
Proof. Let $K$ be the algebraic closure of $\mathbb{Q}$. Let $D$ be an irreducible curve in $Y(K)$, and let $L \subset f^{-1}(D)$ be an arbitrary irreducible curve such that $f(L)$ is dense in $D$. Let $\bar{D}$ and $\bar{L}$ be the projective closures of $D$ and $L$ respectively, and let $\widetilde{L}$ be the smooth projective model of $\bar{L}$. The regular morphism $f: L \rightarrow D$ determines a rational morphism $\widetilde{f}: \widetilde{L} \rightarrow \bar{D}$. Since each rational morphism from a smooth curve into a projective variety is regular and the image of a projective variety under a regular map is closed (see [24]), $\widetilde{f}$ is a regular surjective morphism. Let $v \in \bar{D} \backslash D$ be a point at infinity on $\bar{D}$, and suppose that $w \in \widetilde{f}^{-1}(v)$. The coordinates of the two points $v$ and $w$ generate a finite extension $K_{1} / \mathbb{Q}$. By Chebotarev's density theorem there exist infinitely many primes $p$ such that $K_{1} \subset \mathbb{Q}_{p}$. We choose one such $p$. Then $w \in \widetilde{L}\left(\mathbb{Q}_{p}\right)$ and $v \in \bar{D}\left(\mathbb{Q}_{p}\right)$. Since $w$ is a non-singular point in $\widetilde{L}, w$ has a $p$-adic neighbourhood $W \subset \widetilde{L}\left(\mathbb{Q}_{p}\right)$ such that $W$ is homeomorphic to a disc in $\mathbb{Q}_{p}$ (see [24]; Chapter II). This means that there exists an infinite sequence of elements $w_{i}$ in $W$ such that $w_{i} \in L\left(\mathbb{Q}_{p}\right)$ and $\lim _{i \rightarrow \infty} w_{i}=w$ in the $p$-adic topology. By the continuity of $\widetilde{f}$ we obtain $\lim _{i \rightarrow \infty} \widetilde{f}\left(w_{i}\right)=v$. Since $v \in \bar{D}\left(\mathbb{Q}_{p}\right)$ is a point at infinity, the sequence of elements $f\left(w_{i}\right)=\widetilde{f}\left(w_{i}\right)$ of $D\left(\mathbb{Q}_{p}\right)$ is unbounded. This means that there exists $i$ such that not all coordinates of $f\left(w_{i}\right)$ belong to $\mathbb{Z}_{p}$. This completes the proof.

Proof of Theorem 1. Let $g_{1}, \ldots, g_{s}$ be elements of $\Gamma$ such that the corresponding functions $\tau_{g_{1}}, \ldots, \tau_{g_{s}}$ generate the ring $T(\Gamma)$. Then the projection $\pi: R(\Gamma) \rightarrow X(\Gamma)$ is defined by the formula $\pi(\rho)=\left(\tau_{g_{1}}(\rho), \ldots, \tau_{g_{s}}(\rho)\right)$. By the assumptions of Theorem 1 we have $\operatorname{dim} X^{s}(\Gamma) \geqslant 2$, therefore there exists an irreducible component $Z$ of the closure $\overline{X^{s}(\Gamma)}$ in the Zariski topology such that $\operatorname{dim} Z \geqslant 2$ and $U=Z \cap X^{s}(\Gamma) \neq \varnothing$. Let $Z_{1}$ be the irreducible component of $X(\Gamma)$ containing $Z$. Since $X^{s}(\Gamma)$ is open in $X(\Gamma)$ and $X^{s}(\Gamma) \cap Z_{1}=U$, the set $U$ is dense in $Z_{1}$ in the Zariski topology, and so is also $Z$, that is, $Z=Z_{1}$. Let $p_{i}: Z \rightarrow \mathbb{A}^{1}$ be the projection defined by the formula $p_{i}\left(z_{1}, \ldots, z_{s}\right)=z_{i}$. Since $\operatorname{dim} Z \geqslant 2$, there exists $i$ such that the projection $p_{i}$ is dominant and therefore $p_{i}(U)$ is dense in $\mathbb{A}^{1}$ in the Zariski topology. Hence there exists an integer $n>2$ such that $n \in p_{i}(U)$. Let $Y=p_{i}^{-1}(n) \subset Z$. Then, by the Dimension Theorem $\operatorname{dim} Y \geqslant \operatorname{dim} Z-1 \geqslant 1$ and $Y \cap U \neq \varnothing$. Further, let $X$ be an irreducible component of $\pi^{-1}(Y)$ such that $\pi(X)$ is dense in $Y$. Applying Lemma 2 to the varieties $X$ and $Y$ and the morphism $\pi$ we see that there exists a prime $p$ such that $R\left(\mathbb{Q}_{p}\right)$ contains a representation $\rho$ with the following properties: $\rho$ is irreducible and not all coordinates of the point $\pi(\rho)$ belong to $\mathbb{Z}_{p}$. The latter means that there exists $j$ such that $\tau_{g_{j}}(\rho)=\operatorname{tr} \rho\left(g_{j}\right) \notin \mathbb{Z}_{p}$. Hence $\rho(\Gamma)$ is an unbounded subgroup of $\mathrm{SL}_{2}\left(\mathbb{Q}_{p}\right)$. Moreover, it follows from the construction of $\rho$ that $\tau_{g_{i}}(\rho)=\operatorname{tr} \rho\left(g_{i}\right)=n>2$. Thus, the cyclic subgroup of $\rho(\Gamma)$ generated by $\rho\left(g_{i}\right)$ is infinite and bounded. Hence $\rho(\Gamma)$ is a non-discrete subgroup of $\mathrm{SL}_{2}\left(\mathbb{Q}_{p}\right)$.

Now if $\rho(\Gamma)$ does not lie in the normalizer of a maximal torus, then by Lemma 1 $\rho(\Gamma)$ is dense in $\mathrm{SL}_{2}\left(\mathbb{Q}_{p}\right)$ in the $p$-adic topology; hence $\rho(\Gamma)$ (and therefore also $\Gamma$ ) is a non-trivial free product with amalgamation.

Assume now that $\rho(\Gamma)$ lies in the normalizer of a maximal torus. We claim that there exists an epimorphism $f: \rho(\Gamma) \rightarrow D_{\infty}$, where $D_{\infty}=\langle c, d| d c d^{-1}=c^{-1}$, $\left.d^{2}=1\right\rangle=\mathbb{Z} / 2 \mathbb{Z} * \mathbb{Z} / 2 \mathbb{Z}$ is the infinite dihedral group. Indeed, since $\rho(\Gamma)$ is by 
construction absolutely irreducible and infinite, by [21]; Corollary $2, \rho(\Gamma)$ contains a normal Abelian subgroup $A$ of index 2 and we have $\operatorname{tr} x=0$, that is, $x^{2}=-E$ and $x a x^{-1}=a^{-1}$ for all $x \in G \backslash A, a \in A$. Let $\rho(\Gamma)=A \cup x A$ be a partitioning of $\rho(\Gamma)$ into two cosets. Since $A$ is infinite, there exists an epimorphism $f: A \rightarrow C$, where $C=\langle c\rangle$ is the infinite cyclic subgroup of $D_{\infty}$ generated by $c$. We now set $f(x a)=d f(a)$ for arbitrary $a \in A$. It is easy to verify that we have a well-defined map $f: \rho(\Gamma) \rightarrow D_{\infty}$ and $f$ is an epimorphism. Since $D_{\infty}$ is a non-trivial free product, $\rho(\Gamma)$ (and therefore $\Gamma$ ) is a non-trivial free product with amalgamation. The proof of Theorem 1 is complete.

\section{$\S 2$. Auxiliary results}

In this section we prove several auxiliary results used in the proofs of Theorems 2 and 3. In what follows we shall denote the ring of algebraic integers in $\mathbb{C}$ by $\mathcal{O}$, the group of units in $\mathcal{O}$ by $\mathcal{O}^{*}$, the free group of rank 2 on generators $g$ and $h$ by $F_{2}=\langle g, h\rangle$, and the greatest common divisor of integers $a$ and $b$ by $(a, b)$. If $K \supset L$ is a finite extension of fields and $x \in K$, then we denote the norm of the element $x$ by $N_{K / L}(x)$.

The following lemma characterizes finite-order elements of $\mathrm{SL}_{2}(\mathbb{C})$.

Lemma 3. Suppose that $m \in \mathbb{Z}, m>2$, and that $X \in \mathrm{SL}_{2}(\mathbb{C}), X \neq \pm E$. Then $X^{m}=E$ if and only if $\operatorname{tr} X=\varepsilon+\varepsilon^{-1}$, where $\varepsilon^{m}=1, \varepsilon \neq \pm 1$ (in other words, if and only if $\operatorname{tr} X=2 \cos (2 r \pi / m)$ for some $r \in\{1, \ldots, m-1\})$. In particular, if $\operatorname{tr} X=0$, then $X^{2}=-E$.

Proof. If $X^{m}=E$, then the assertion is obvious. If $\operatorname{tr} X=\varepsilon+\varepsilon^{-1}$, then $\varepsilon$ and $\varepsilon^{-1}$ are the eigenvalues of $X$. Hence $X$ is conjugate to the matrix $\operatorname{diag}\left(\varepsilon, \varepsilon^{-1}\right)$, that is, $X^{m}=E$, as required.

Obviously, the representation variety $R\left(F_{2}\right)$ of the free group $F_{2}=\langle g, h\rangle$ is $\mathrm{SL}_{2}(\mathbb{C}) \times \mathrm{SL}_{2}(\mathbb{C})$. It is known that the ring $T\left(F_{2}\right)$ is generated by the functions $\tau_{g}, \tau_{h}, \tau_{g h}$ (see [12], [16], [17]). For $u$ in $F_{2}$ the function $\tau_{u}$ is usually called the Fricke character of the element $u$.

Lemma 4. For all $\alpha, \beta, \gamma \in \mathbb{C}$ there exist matrices $A$ and $B$ in $\mathrm{SL}_{2}(\mathbb{C})$ such that $\tau_{g}(A, B)=\operatorname{tr} A=\alpha, \tau_{h}(A, B)=\operatorname{tr} B=\beta, \tau_{g h}(A, B)=\operatorname{tr} A B=\gamma$.

This lemma can be easily proved by straightforward computation.

In particular, Lemma 4 yields the equality $X\left(F_{2}\right)=\pi\left(R\left(F_{2}\right)\right)=\mathbb{A}^{3}$. Moreover, the functions $\tau_{g}, \tau_{h}, \tau_{g h}$ are algebraically independent over $\mathbb{C}$ and for each $u \in F_{2}$ we have

$$
\tau_{u}=Q_{u}\left(\tau_{g}, \tau_{h}, \tau_{g h}\right),
$$

where $Q_{u} \in \mathbb{Z}[x, y, z]$ is a uniquely defined polynomial with integer coefficients. The polynomial $Q_{u}$ is usually called the Fricke polynomial of the element $u$. The following relations for Fricke characters are consequences of the relations between the traces of arbitrary matrices in $\mathrm{SL}_{2}(\mathbb{C})$ :
(1) $\tau_{u^{-1}}=\tau_{u}$;
(2) $\tau_{u v}=\tau_{v u}$
(3) $\tau_{v u v^{-1}}=\tau_{u}$;
(4) $\tau_{u v}=\tau_{u} \tau_{v}-\tau_{u v^{-1}}$. 
We require now more detailed information on the Fricke polynomials (see [25]). Consider the polynomials $P_{n}(\lambda)$ satisfying the initial conditions

$$
P_{-1}(\lambda)=0, \quad P_{0}(\lambda)=1
$$

and the recursive relation

$$
P_{n}(\lambda)=\lambda P_{n-1}(\lambda)-P_{n-2}(\lambda) .
$$

If $n<0$, then we set $P_{n}(\lambda)=-P_{|n|-2}(\lambda)$. The degree of $P_{n}(\lambda)$ is $n$ if $n>0$ and $|n|-2$ if $n<0$. It can be easily verified by induction on $n$ that

$$
P_{n}(2 \cos (\varphi))=\frac{\sin ((n+1) \varphi)}{\sin (\varphi)} .
$$

It follows from (2) that the polynomial $P_{n}(\lambda), n \geqslant 1$, has $n$ zeros described by the formula

$$
\lambda_{n, k}=2 \cos \left(\frac{k \pi}{n+1}\right), \quad k=1,2, \ldots, n .
$$

Moreover, it is easy to verify by induction that for $n>0$ we have

$$
\begin{aligned}
P_{2 n}(\lambda) & =\lambda^{2 n}+\cdots+(-1)^{n}, \\
P_{2 n-1}(\lambda) & =\lambda\left(\lambda^{2 n-2}+\cdots+(-1)^{n-1} n\right) .
\end{aligned}
$$

Further, let $w=g^{\alpha_{1}} h^{\beta_{1}} \ldots g^{\alpha_{s}} h^{\beta_{s}}$ be a cyclically reduced word in $F_{2}$ and set $x=\tau_{g}$, $y=\tau_{h}, z=\tau_{g h}$. We shall treat the Fricke polynomial $Q_{w}(x, y, z)$ as a polynomial in $z$. Let

$$
Q_{w}(x, y, z)=M_{n}(x, y) z^{n}+M_{n-1}(x, y) z^{n-1}+\cdots+M_{0}(x, y) .
$$

Lemma 5 [25]. The degree of the Fricke polynomial $Q_{w}(x, y, z)$ with respect to $z$ is equal to $s$, that is, the number of the blocks of the form $g^{\alpha_{i}} h^{\beta_{i}}$ in $w$. The leading coefficient $M_{s}(x, y)$ of $Q_{w}(x, y, z)$ has the following form:

$$
M_{s}(x, y)=\prod_{i=1}^{s} P_{\alpha_{i}-1}(x) P_{\beta_{i}-1}(y) .
$$

The following lemma plays an important role in the proofs of Theorems 2 and 3.

Lemma 6. Let $\Gamma=\left\langle a, b \mid a^{n}=R^{m}(a, b)=1\right\rangle$, where $n=0$ or $n \geqslant 2, m \geqslant 2$, and $R(a, b)$ is a cyclically reduced word containing $b$ in the free group on $a$ and $b$. Assume that there exist matrices $A$ and $B$ in $\mathrm{SL}_{2}(\mathbb{C})$ such that $\operatorname{tr} A=\alpha=2 \cos (t \pi / n)$ for some $t \in\{1, \ldots, n-1\}$ and $\operatorname{tr} R(A, B)=Q_{R}(\alpha, y, z)=c$, where $Q_{R}$ is the Fricke polynomial of the element $R(g, h)$ of $F_{2}, c=2 \cos (r \pi / m)$ for some $r \in\{1, \ldots, m-1\}, y=\operatorname{tr} B$, and $z=\operatorname{tr} A B$. Let $H=\langle A, B\rangle$ be the group generated by the matrices $A$ and $B$. Assume that the following two conditions hold:

(1) there exists a unipotent (or finite-order) element $W$ of $H$ of the form $A^{\alpha_{1}} B^{\beta_{1}} \cdots A^{\alpha_{s}} B^{\beta_{s}}$ such that $\alpha_{i}, \beta_{i} \neq 0$ for $i=1, \ldots, s$ and $l=\sum_{i=1}^{s} \beta_{i} \neq 0$;

(2) there exists an element $h$ of $H$ such that $\operatorname{tr} h \notin \mathcal{O}$.

Then $\Gamma$ is a non-trivial free product with amalgamation. 
Furthermore, suppose that the following condition holds instead of condition (1):

$\left(1^{\prime}\right)$ the matrix $B$ has finite order, that is, $\operatorname{tr} B=2 \cos \left(k_{1} \pi / k\right)$ for some $k \geqslant 2$ and $k_{1} \in\{1, \ldots, k-1\}$.

Then the group $\Gamma_{1}=\left\langle a, b \mid a^{n}=b^{k v}=R^{m}(a, b)=1\right\rangle$ is a non-trivial free product with amalgamation for each integer $v$.

The proof of this lemma is based on Bass's classification of finitely generated subgroups of $\mathrm{SL}_{2}(\mathbb{C})[26]$.

Proposition 1 [26]. Let $H$ be a finitely generated subgroup of $\mathrm{GL}_{2}(\mathbb{C})$. Then one of the following cases must occur:

(1) there exists an epimorphism $f: H \rightarrow \mathbb{Z}$ such that $f(u)=0$ for all unipotent elements $u$ of $H$;

(2) $\operatorname{tr} h \in \mathcal{O}$ for each element $h$ of $H$;

(3) $H$ is a non-trivial free product with amalgamation.

Proof of Lemma 6. It is easy to see that $H$ does not satisfy conditions (1) and (2) in Proposition 1. For let $f: H \rightarrow \mathbb{Z}$ be an epimorphism such that $f(z)=0$ for each unipotent element $z$ of $H$. Then $f(A)=0$, because $A^{2 n}=E$ by Lemma 3 . Furthermore, $f(u)=l f(B)=0$, so that $f(B)=0$ because $u$ is by assumption either unipotent or of finite order and $l \neq 0$. Thus, $f(H)=\{0\}$, which is a contradiction. By assumption $H$ does not satisfy condition (2) in Proposition 1 either. Hence $H$ is a non-trivial free product with amalgamation, that is, $H=H_{1} *_{F} H_{2}$, where $H_{1} \neq F \neq H_{2}$. Let $\bar{A}, \bar{B}, \bar{H}, \bar{H}_{1}, \bar{H}_{2}$, and $\bar{F}$ be the images of $A, B, H, H_{1}, H_{2}$, and $F$ in $\mathrm{PSL}_{2}(\mathbb{C})$, respectively. If $-E \notin H$, then $H$ and $\bar{H}$ are isomorphic. If $-E \in H$, then $-E$ belongs to the centre of $H$, therefore $-E \in F$. In all these cases $\bar{H}_{1} \neq \bar{F} \neq \bar{H}_{2}$ and therefore $\bar{H}=\bar{H}_{1} * \bar{F} \bar{H}_{2}$ is a non-trivial free product with amalgamation. By Lemma 3 , the conditions $\operatorname{tr} A=\alpha$ and $Q_{R}(\alpha, y, z)=c$ yield the equality $A^{2 n}=R^{2 m}(A, B)=E$. Hence $\bar{A}^{n}=R^{m}(\bar{A}, \bar{B})=1$ in $\mathrm{PSL}_{2}(\mathbb{C})$. Thus, $\bar{H}$ is an epimorphic image of $\Gamma$ and therefore $\Gamma$ is also a non-trivial free product with amalgamation.

Next, if we replace the condition (1) by $\left(1^{\prime}\right)$, then again $\bar{H}$ is a non-trivial free product with amalgamation. Moreover, $\bar{A}^{n}=\bar{B}^{k}=R^{m}(\bar{A}, \bar{B})=1$ in $\operatorname{PSL}_{2}(\mathbb{C})$. Hence $\bar{H}$ is an epimorphic image of $\Gamma_{1}$. Thus, $\Gamma_{1}$ is a non-trivial free product with amalgamation, which completes the proof of Lemma 6 .

Lemma 7. (1) Let $r, s$ be integers such that $s \geqslant 3$ and $(r, s)=1$. Then $\cos (r \pi / s) \notin \mathcal{O}$.

(2) For $s \in \mathbb{Z}, s \geqslant 1$, assume that $r \not \equiv 0(\bmod 2 s+1)$. Then $2 \cos (r \pi /(2 s+1)) \in \mathcal{O}^{*}$.

(3) Suppose that $u \in \mathbb{Z}, u \neq 0$, let $p$ be a prime, and let $\varepsilon$ be a primitive root of unity of degree $4 p u$. Also set

$$
x_{r}=2 \cos \left(\frac{r \pi}{2 p u}\right), \quad y_{r}=2 \sin \left(\frac{r \pi}{2 p u}\right), \quad K=\mathbb{Q}(\varepsilon) .
$$

Then there exist $r, r_{1} \not \equiv 0(\bmod p)$ such that $p$ divides both integers $N_{K / \mathbb{Q}}\left(x_{r}\right)$ and $N_{K / \mathbb{Q}}\left(y_{r_{1}}\right)$. In particular, $x_{r}, y_{r_{1}} \notin \mathcal{O}^{*}$.

(4) Suppose that $u, c \in \mathbb{Z},|u| \geqslant 2, c \neq 0$, and let $p$ be a prime not dividing $c$. Further, set $x_{0}=-2 \cos (\pi / u), x_{r}=2 \cos (r \pi /(p u))$. Then there exists $r \not \equiv 0$ $(\bmod p)$ such that $c /\left(x_{r}-x_{0}\right) \notin \mathcal{O}$. 
(5) Let $p$ be a prime, $p>2$. Then $\sin \left(r \pi / p^{s}\right) \notin \mathcal{O}^{*}$ whenever $r \not \equiv 0(\bmod p)$ and $s \geqslant 1$.

(6) Assume that $t \geqslant 1$. Then $2 \sin \left(r \pi / 2^{t}\right) \notin \mathcal{O}^{*}$ for each odd $r$.

Proof. (1) Assume that $\cos (r \pi / s) \in \mathcal{O}$, so that $\cos (d r \pi / s) \in \mathcal{O}$ for each $d$ in $\mathbb{Z}$. By assumption $(r, s)=1$, therefore for each integer $l$ there exists $d$ such that $d r \equiv l(\bmod s)$. Hence for each integer $l$ we have $\cos (l \pi / s) \in \mathcal{O}$. By $(3)$ the polynomial $P_{s-1}(\lambda)$ has the zeros $2 \cos (l \pi / s), l=1, \ldots, s-1$, therefore $P_{s-1}(2 \lambda)$ has the zeros $\cos (l \pi / s), l=1, \ldots, s-1$. If $s=2 s_{1}+1$ is odd, then it follows from (4) that $P_{2 s_{1}}(2 \lambda)=2^{2 s_{1}} \lambda^{2 s_{1}}+\cdots+(-1)^{s_{1}}$. Since $1 / 2^{2 s_{1}} \notin \mathbb{Z}$, the polynomial $P_{2 s_{1}}(2 \lambda)$ has a zero not belonging to $\mathcal{O}$, that is, there exists $l$ such that $\cos (l \pi / s) \notin \mathcal{O}$, which is a contradiction. If $s=2 s_{1}$ is even, then it follows from (4) that $P_{2 s_{1}-1}(2 \lambda)=$ $2 \lambda\left(2^{2 s_{1}-2} \lambda^{2 s_{1}-2}+\cdots+(-1)^{s_{1}-1} s_{1}\right)$. By assumption $s \geqslant 3$, therefore $s_{1} \geqslant 2$. Hence $s_{1} / 2^{2 s_{1}-2} \notin \mathbb{Z}$ and $P_{2 s_{1}-1}(2 \lambda)$ has a zero not belonging to $\mathcal{O}$. Again, this is a contradiction, which proves part (1).

(2) By (3) and (4) the quantity $2 \cos (r \pi /(2 s+1))$ is a zero of the polynomial $P_{2 s}(\lambda)=\lambda^{2 s}+\cdots+(-1)^{s}$ and therefore belongs to $\mathcal{O}^{*}$.

(3) Since $y_{r}=2 \cos ((p u-r) \pi /(2 p u))=x_{p u-r}$, it is sufficient to prove the assertion for $x_{r}$. Let $u=p^{f} u^{\prime}$, where $f \geqslant 0, p \nmid u^{\prime}$, and let $r=r_{1} u^{\prime}$, where $p \nmid r_{1}$. Then $x_{r}=2 \cos \left(r_{1} \pi /\left(2 p^{f+1}\right)\right)$. By (3) and (4) the polynomial

$$
P_{2 p^{f+1}-1}(\lambda)=\lambda\left(\lambda^{2 p^{f+1}-2}+\cdots+(-1)^{p^{f+1}-1} p^{f+1}\right)
$$

has the zeros $2 \cos \left(r^{\prime} \pi /\left(2 p^{f+1}\right)\right), r^{\prime}=1, \ldots, 2 p^{f+1}-1$, and the polynomial

$$
P_{2 p^{f}-1}(\lambda)=\lambda\left(\lambda^{2 p^{f}-2}+\cdots+(-1)^{p^{f}-1} p^{f}\right)
$$

has the zeros $2 \cos \left(r^{\prime} \pi /\left(2 p^{f}\right)\right), r^{\prime}=1, \ldots, 2 p^{f}-1$. Hence $P_{2 p_{-1}^{f}}(\lambda)$ divides $P_{2 p^{f+1}-1}(\lambda)$, that is,

$$
P_{2 p^{f+1}-1}(\lambda)=P_{2 p^{f}-1}(\lambda) F(\lambda),
$$

where $F(\lambda)$ is easily seen to be a polynomial of degree $2\left(p^{f+1}-p^{f}\right)$ with constant term $p$ and leading coefficient 1 . The zeros of $F(\lambda)$ have the form $2 \cos \left(r^{\prime} \pi /\left(2 p^{f+1}\right)\right)$, $r^{\prime} \not \equiv 0(\bmod p)$. It is easy to see that there exists $r_{1} \not \equiv 0(\bmod p)$ such that $N_{K / \mathbb{Q}}\left(2 \cos \left(r_{1} \pi /\left(2 p^{f+1}\right)\right)\right)= \pm p^{s}$ for some $s \geqslant 1$, as required.

(4) Note that

$$
x_{r}-x_{0}=2 \cos \left(\frac{r \pi}{p u}\right)+2 \cos \left(\frac{\pi}{u}\right)=\left(2 \cos \left(\frac{(r+p) \pi}{2 p u}\right)\right)\left(2 \cos \left(\frac{(r-p) \pi}{2 p u}\right)\right) .
$$

Hence it is sufficient to show that for some $r \not \equiv 0(\bmod p)$ we have $c / \alpha_{r} \notin \mathcal{O}$, where $\alpha_{r}=2 \cos ((r+p) \pi /(2 p u))$. Let $K_{r}=\mathbb{Q}\left(\alpha_{r}\right)$ and $d_{r}=\left[K_{r}: \mathbb{Q}\right]$. By part (3), proved above, there exists $r \not \equiv 0(\bmod p)$ such that $p$ divides $N_{K_{r} / \mathbb{Q}}\left(\alpha_{r}\right)$. Hence

$$
N_{K_{r} / \mathbb{Q}}\left(\frac{c}{\alpha_{r}}\right)=\frac{c^{d_{r}}}{N_{K_{r} / \mathbb{Q}}\left(\alpha_{r}\right)} \notin \mathbb{Z}
$$

because $p \nmid c$ by assumption. Hence $c / \alpha_{r} \notin \mathcal{O}$, as required. 
(5) Note that $1 / \sin \left(r \pi / p^{s}\right)=2 /\left(2 \cos \left(\left(p^{s}-2 r\right) \pi /\left(2 p^{s}\right)\right)\right)$. It follows from our proof of part (4) that there exists $r_{0} \not \equiv 0(\bmod p)$ such that

$$
\frac{1}{\sin \left(r_{0} \pi / p^{s}\right)}=\frac{2}{2 \cos \left(\left(p^{s}-2 r_{0}\right) \pi /\left(2 p^{s}\right)\right)} \notin \mathcal{O}
$$

We now claim that for each $r \not \equiv 0(\bmod p)$ we have $\sin \left(r \pi / p^{s}\right) \notin \mathcal{O}$. Assume the contrary. Suppose that $1 / \sin \left(r \pi / p^{s}\right) \in \mathcal{O}$ for some $r$ with $(r, p)=1$. Since $\left(p, r_{0}\right)=1$, there exists $d$ such that $r \equiv d r_{0}\left(\bmod p^{s}\right)$. Hence by $(2)$ we obtain

$$
P_{d}\left(2 \cos \left(\frac{r_{0} \pi}{p^{s}}\right)\right)=\frac{\sin \left(d r_{0} \pi / p^{s}\right)}{\sin \left(r_{0} \pi / p^{s}\right)}= \pm \frac{\sin \left(r \pi / p^{s}\right)}{\sin \left(r_{0} \pi / p^{s}\right)},
$$

which immediately shows that

$$
\frac{1}{\sin \left(r_{0} \pi / p^{s}\right)}= \pm \frac{1}{\sin \left(r \pi / p^{s}\right)} P_{d}\left(2 \cos \left(\frac{r_{0} \pi}{p^{s}}\right)\right) \in \mathcal{O}
$$

which is a contradiction.

(6) For $t=1$ the assertion is obvious. Assume that $t>1$. By part (3) there exists an odd $r_{0}$ such that $2 \sin \left(r_{0} \pi / 2^{t}\right) \notin \mathcal{O}^{*}$. We claim that for each odd $r$ we have $2 \sin \left(r \pi / 2^{t}\right) \notin \mathcal{O}^{*}$. Assume the contrary, and suppose that $2 \sin \left(r \pi / 2^{t}\right) \in \mathcal{O}^{*}$ for some odd $r$. Obviously, there exists an integer $d$ such that $r \equiv d r_{0}\left(\bmod 2^{t}\right)$. Then by (2) we obtain

$$
P_{d}\left(2 \cos \left(\frac{r_{0} \pi}{2^{t}}\right)\right)= \pm \frac{2 \sin \left(r \pi / 2^{t}\right)}{2 \sin \left(r_{0} \pi / 2^{t}\right)} .
$$

The last equality yields the inclusion $2 \sin \left(r_{0} \pi / 2^{t}\right) \in \mathcal{O}^{*}$, which is the contradiction completing the proof of Lemma 7.

Lemma 8. (1) Suppose that $s, t \geqslant 0$. Then

$$
P_{s}(\lambda) P_{t}(\lambda)=\sum_{i=0}^{t} P_{s-t+2 i}(\lambda)
$$

(2) The polynomial $P_{s}(\lambda)-P_{s-1}(\lambda)$ has the zeros $\lambda_{r}=2 \cos ((2 r+1) \pi /(2 s+1))$, $r \in\{0,1, \ldots, s-1\}$.

(3) If $\gamma=2 \cos (2 r \pi /(2 s+1))$, where $s \geqslant 1, r \in\{1, \ldots, s\}$ and $(r, 2 s+1)=1$, then $P_{s}(\gamma)-P_{s-1}(\gamma) \notin \mathcal{O}^{*}$.

(4) If $\gamma=2 \cos ((2 r+1) \pi /(2 s)) \neq 0$, where $s \geqslant 2$ and $(s, 2 r+1)=1$, then $0 \neq P_{s-1}(\gamma) \notin \mathcal{O}^{*}$.

(5) Suppose that $\gamma \in \mathcal{O}$. Assume that $\gamma$ is not equal to $2 \cos (r \pi / s)$, where $r, s \in \mathbb{Z}$. Then there exists an integer $l>0$ such that $P_{l}(\gamma) \notin \mathcal{O}^{*}$.

Proof. (1) We fix $s$ and proceed by induction on $t$. If $t=0$, then we have $P_{s}(\lambda) P_{0}(\lambda)=P_{s}(\lambda)$. If $t=1$, then $P_{s}(\lambda) P_{1}(\lambda)=P_{s}(\lambda) \lambda=P_{s+1}(\lambda)+P_{s-1}(\lambda)$ 
by definition. Further, by induction we obtain

$$
\begin{aligned}
P_{s}(\lambda) P_{t}(\lambda) & =P_{s}(\lambda)\left(\lambda P_{t-1}(\lambda)-P_{t-2}(\lambda)\right) \\
& =\lambda \sum_{i=0}^{t-1} P_{s-t+1+2 i}(\lambda)-\sum_{i=0}^{t-2} P_{s-t+2+2 i}(\lambda) \\
& =\sum_{i=0}^{t-1}\left(P_{s-t+2+2 i}(\lambda)+P_{s-t+2 i}(\lambda)\right)-\sum_{i=0}^{t-2} P_{s-t+2+2 i}(\lambda) \\
& =P_{s+t}(\lambda)+\sum_{i=0}^{t-1} P_{s-t+2 i}(\lambda)=\sum_{i=0}^{t} P_{s-t+2 i}(\lambda)
\end{aligned}
$$

as required.

(2) Bearing in mind (2) we see that

$P_{s}\left(\lambda_{r}\right)-P_{s-1}\left(\lambda_{r}\right)=\frac{\sin ((2 r+1)(s+1) \pi /(2 s+1))-\sin ((2 r+1) s \pi /(2 s+1))}{\sin ((2 r+1) \pi /(2 s+1))}=0$.

(3) Using (2) we obtain

$$
\frac{1}{P_{s}(\gamma)-P_{s-1}(\gamma)}=\frac{\sin (2 r \pi /(2 s+1))}{2 \sin (r \pi /(2 s+1)) \cos (r \pi)}= \pm \cos \left(\frac{r \pi}{2 s+1}\right) \notin \mathcal{O}
$$

by Lemma 7(1).

(4) Using (2) again, we obtain

$$
\frac{1}{P_{s-1}(\gamma)}=\frac{\sin ((2 r+1) \pi /(2 s))}{\sin ((2 r+1) \pi / 2)}=(-1)^{r} \cos \left(\frac{(s-2 r-1) \pi}{2 s}\right) \notin \mathcal{O}
$$

by Lemma 7(1).

(5) Since the polynomial $P_{l}(\lambda)$ has by (3) the zeros $2 \cos (r \pi /(l+1)), r=1, \ldots, l$, one can write: $P_{l}(\gamma)=\prod_{r=1}^{l}(\gamma-2 \cos (r \pi /(l+1)))$. Hence it is sufficient to prove that $\gamma-\left(\varepsilon+\varepsilon^{-1}\right) \notin \mathcal{O}^{*}$, where $\varepsilon \neq \pm 1$ is some root of unity. Let $f(\lambda)$ be a polynomial for $\gamma$ irreducible over $\mathbb{Q}, K_{0}$ the splitting field of $f(\lambda)$, and set $K_{1}=K_{0}\left(x_{0}\right)$, where $x_{0}$ is a root of the equation $x+x^{-1}=\gamma$. Let $Z_{1}$ be the integral closure of $\mathbb{Z}$ in $K_{1}$ and let $p$ be an odd prime. Let $\mathfrak{p}_{1}$ be a prime ideal in $Z_{1}$ lying over $(p)$. Then $k_{1}=Z_{1} / \mathfrak{p}_{1} \supset \mathbb{Z} / p \mathbb{Z}=k$ is a finite extension of fields. We have $x_{0}, y_{0} \in Z_{1}$. Let $\bar{x}_{0}$ and $\bar{\gamma}$ be the images of $x_{0}$ and $\gamma$ in $k_{1}$, respectively. Then the following equality holds:

$$
\bar{x}_{0}+\bar{x}_{0}^{-1}=\bar{\gamma} .
$$

Let $l=\left|k_{1}^{*}\right|$ be the order of the multiplicative group of $k_{1}$. Then $\bar{x}_{0}^{l}=1$ in $k_{1}$. Consider the field $K_{2}=K_{1}(\xi)$, where $\xi$ is a primitive root of unity of degree $l$ in $\mathbb{C}$. Let $Z_{2}$ be the integral closure of $Z_{1}$ in $K_{2}, \mathfrak{p}_{2}$ a prime ideal in $Z_{2}$ lying above $\mathfrak{p}_{1}$, and set $k_{2}=Z_{2} / \mathfrak{p}_{2} \supset k_{1}$. We denote by $\Delta$ the group of roots of unity of degree $l$ in $K_{2}$ and by $\bar{\Delta}$ its image in $k_{2}$. We claim that $\bar{\Delta}=k_{1}^{*}$. Assume that, on the contrary, $\bar{\Delta} \neq k_{1}^{*}$. Then for some integer $r, 0<r<l$, we have $\bar{\xi}^{r}=1$, where $\bar{\xi}$ is 
the image of $\xi$ in $k_{2}$. This means that $\xi^{r}=1+y$, where $y \in \mathfrak{p}_{2}$. Then $(1+y)^{l}=1$, that is, $1+C_{l}^{1} y+\cdots+C_{l}^{l} y^{l}=1$, where $C_{l}^{i}$ is the corresponding binomial coefficient. Hence $y\left(l+y y_{1}\right)=0$, where $y_{1}=C_{l}^{2} y+\cdots+C_{l}^{l} y^{l-1}$. Since $y \neq 0$, it follows that $l \in \mathfrak{p}_{2} \cap \mathbb{Z}=(p)$. However, $l=\left|k_{1}^{*}\right|=p^{t}-1$ for some $t$, which is a contradiction. Hence there exists a root of unity $\varepsilon$ of degree $l$ such that $\bar{\varepsilon}=\bar{x}_{0}$. This means that $\gamma-\left(\varepsilon+\varepsilon^{-1}\right) \in \mathfrak{p}_{2}$ and therefore $\gamma-\left(\varepsilon+\varepsilon^{-1}\right)$ is not a unit in the ring $\mathcal{O}$. This completes the proof of Lemma 8.

Lemma 9. Let $F_{2}=\langle g, h\rangle$ be the free group on generators $g$ and $h$. Set $x=\tau_{g}$, $y=\tau_{h}, z=\tau_{g h}$, and $t=\tau_{g h g^{-1} h^{-1}}$. Then the following assertions hold.

(1) $t=x^{2}+y^{2}+z^{2}-x y z-2$.

(2) Suppose that $R=g h\left(g h g^{-1} h^{-1}\right)^{s}$. Then

$$
\tau_{R}=\left(P_{s}(t)-P_{s-1}(t)\right) z .
$$

(3) Suppose that $T=(g h)^{-1}\left(g h g^{-1} h^{-1}\right)^{s}(g h)^{2}\left(g h g^{-1} h^{-1}\right)^{s}$. Then

$$
\tau_{T}=(t-2) P_{s-1}(t)^{2} z^{3}+\left(2-P_{2 s-1}(t)+P_{2 s-2}(t)\right) z .
$$

Proof. (1) One can prove the equality in question by straightforward computation using relations (1) (see [16]).

(2) Let $u$ and $v$ be arbitrary elements of $F_{2}$. Then it is easy to show, using induction and relations (1), that the following equality holds for all integers $p$ and $q$ :

$$
\tau_{u^{p} v^{q}}=P_{p-1}\left(\tau_{u}\right) P_{q-1}\left(\tau_{v}\right) \tau_{u v}-P_{p-2}\left(\tau_{u}\right) P_{q}\left(\tau_{v}\right)-P_{p}\left(\tau_{u}\right) P_{q-2}\left(\tau_{v}\right) .
$$

We now set $u=g h$ and $v=g h g^{-1} h^{-1}$. Then

$$
\tau_{u}=z, \quad \tau_{v}=t, \quad \tau_{u v}=\tau_{g h\left(g h g^{-1} h^{-1}\right)}=z t-\tau_{g^{-1} h^{-1}}=z(t-1) .
$$

Hence

$$
\begin{aligned}
\tau_{u v^{s}} & =P_{s-1}\left(\tau_{v}\right) \tau_{u v}-P_{s-2}\left(\tau_{v}\right) \tau_{u}=P_{s-1}(t)(t-1) z-P_{s-2}(t) z \\
& =z\left(t P_{s-1}(t)-P_{s-1}(t)-P_{s-2}(t)\right)=z\left(P_{s}(t)+P_{s-2}(t)-P_{s-1}(t)-P_{s-2}(t)\right) \\
& =z\left(P_{s}(t)-P_{s-1}(t)\right) .
\end{aligned}
$$

(3) Let $u$ and $v$ be as above. Then using relations (1) and (8) we obtain

$$
\begin{aligned}
\tau_{u^{-1} v^{s}} & =\tau_{u^{-1}} \tau_{v^{s}}-\tau_{u v^{s}}=z\left(P_{s}(t)-P_{s-2}(t)\right)-z\left(P_{s}(t)-P_{s-1}(t)\right) \\
& =z\left(P_{s-1}(t)-P_{s-2}(t)\right) ; \\
\tau_{u^{2} v^{s}} & =\tau_{u} \tau_{u v^{s}}-\tau_{v^{s}}=z^{2}\left(P_{s}(t)-P_{s-1}(t)\right)-P_{s}(t)+P_{s-2}(t) ; \\
\tau_{u^{3}} & =z^{3}-3 z .
\end{aligned}
$$

Hence

$$
\begin{gathered}
\tau_{u^{-1} v^{s} u^{2} v^{s}=} \tau_{u^{-1} v^{s}} \tau_{u^{2} v^{s}}-\tau_{u^{3}}=z^{3}\left(\left(P_{s}(t)-P_{s-1}(t)\right)\left(P_{s-1}(t)-P_{s-2}(t)\right)-1\right) \\
+z\left(3-\left(P_{s-1}(t)-P_{s-2}(t)\right)\left(P_{s}(t)-P_{s-2}(t)\right)\right) .
\end{gathered}
$$


We simplify the last equation using (7). We consider the coefficient of $z^{3}$ first:

$$
\begin{aligned}
\left(P_{s}(t)\right. & \left.-P_{s-1}(t)\right)\left(P_{s-1}(t)-P_{s-2}(t)\right)-1 \\
& =P_{s}(t) P_{s-1}(t)+P_{s-1}(t) P_{s-2}(t)-P_{s}(t) P_{s-2}(t)-P_{s-1}(t)^{2}-1 \\
& =P_{s-1}(t)\left(P_{s}(t)+P_{s-2}(t)\right)-\sum_{i=1}^{s-1} P_{2 i}(t)-P_{0}(t)-P_{s-1}(t)^{2} \\
& =t P_{s-1}(t)^{2}-2 P_{s-1}(t)^{2}=(t-2) P_{s-1}(t)^{2} .
\end{aligned}
$$

Next we consider the coefficient of $z$ :

$$
\begin{aligned}
3- & \left(P_{s-1}(t)-P_{s-2}(t)\right)\left(P_{s}(t)-P_{s-2}(t)\right) \\
& =3-P_{s}(t) P_{s-1}(t)+P_{s-1}(t) P_{s-2}(t)+P_{s}(t) P_{s-2}(t)-P_{s-2}(t)^{2} \\
& =3-\sum_{i=1}^{s} P_{2 i-1}(t)+\sum_{i=1}^{s-1} P_{2 i-1}(t)+\sum_{i=1}^{s-1} P_{2 i}(t)-\sum_{i=0}^{s-2} P_{2 i}(t) \\
& =2-P_{2 s-1}(t)+P_{2 s-2}(t) .
\end{aligned}
$$

This completes the proof of Lemma 9.

At the end of $\S 2$ we show how one can deduce Corollary 1 from Theorem 1 . This will give us another proof of Conjecture 1 . Let $\Gamma=\left\langle a, b \mid R^{m}(a, b)=1\right\rangle$, where $m \geqslant 2, R(a, b)=a^{u_{1}} b^{v_{1}} \cdots a^{u_{s}} b^{v_{s}}, u_{i}, v_{i} \neq 0, s \geqslant 1$, and $R(a, b)$ is not a proper power.

We consider the case $m \geqslant 3$ first. We claim that $\operatorname{dim} X^{s}(\Gamma) \geqslant 2$. Then Theorem 1 immediately yields that $\Gamma$ is a non-trivial free product with amalgamation. In the character variety $X\left(F_{2}\right)=\mathbb{A}^{3}$ of the free group $F_{2}=\langle g, h\rangle$ we consider the hypersurface $V$ defined by the equation

$$
\tau_{R(g, h)}(x, y, z)=2 \cos \left(\frac{2 \pi}{m}\right),
$$

where $x=\tau_{g}, y=\tau_{h}$, and $z=\tau_{g h}$. By Lemma 5 one can write (9) in the following form:

$$
f(x, y, z)=M_{s}(x, y) z^{s}+\cdots+M_{0}(x, y)-2 \cos \left(\frac{2 \pi}{m}\right)=0 .
$$

We claim that $V \subset X(\Gamma)$. For let $v=\left(x_{0}, y_{0}, z_{0}\right) \in V$ and let $A$ and $B$ be matrices in $\mathrm{SL}_{2}(\mathbb{C})$ such that $\operatorname{tr} A=x_{0}, \operatorname{tr} B=y_{0}$, and $\operatorname{tr} A B=z_{0}$. Then by Lemma 3 we obtain the equality $R^{m}(A, B)=E$. Hence the pair of matrices $(A, B)$ determines a representation $\rho$ of $\Gamma$ into $\mathrm{SL}_{2}(\mathbb{C})$. Moreover, the range of $\rho$ in $X(\Gamma)$ coincides with $v$, so that $v \in X(\Gamma)$. Further, let $V_{1}, \ldots, V_{r}$ be the irreducible components of $V$. It is easy to see (cf. [24]) that $\operatorname{dim} V_{i}=2$ for each $i$. It remains to show that $V \cap X^{s}(\Gamma) \neq \varnothing$. Assume the contrary. Then all representations corresponding to points in $V$ are reducible. This means that the regular function $\tau_{g h g^{-1} h^{-1}}-2$ is identically equal to 0 on $V$. Hence by Lemma 9(1) we obtain

$$
g(x, y, z)=x^{2}+y^{2}+z^{2}-x y z-4 \equiv 0
$$


on $V$. Thus,

$$
f(x, y, z)=C g(x, y, z)^{d},
$$

where $C$ is a constant distinct from zero and $d \geqslant 1$.

If we have $\left|u_{i}\right| \geqslant 2$ or $\left|v_{i}\right| \geqslant 2$ for some $i$, then the leading coefficient $M_{s}(x, y)$ in (10) is not a constant, by Lemma 5 , and equality (11) is impossible.

Now suppose that $\left|u_{i}\right|=\left|v_{i}\right|=1$ for $i=1, \ldots, s$. First of all, if for some $i$ we have $u_{i}=u_{i+1}$ or $v_{i}=v_{i+1}\left(u_{1}=u_{s}\right.$ or $v_{1}=v_{s}$ for $\left.i=s\right)$, then we can consider other generators of $\Gamma$. Assume for definiteness that $u_{1}=u_{2}$. We set $a_{1}=a^{u_{1}} b^{v_{1}}$, $b_{1}=b$. Then $\Gamma=\left\langle a_{1}, b_{1} \mid R_{1}^{m}\left(a_{1}, b_{1}\right)=1\right\rangle$, where $R_{1}^{m}\left(a_{1}, b_{1}\right)=a_{1}^{u_{1}^{\prime}} b_{1}^{v_{1}^{\prime}} \cdots a_{1}^{u_{r}^{\prime}} b_{1}^{v_{r}^{\prime}}$, where $u_{i}^{\prime}, v_{i}^{\prime} \neq 0, r \geqslant 1$, and $u_{1}^{\prime} \geqslant 2$. We considered this case above.

We can thus assume without loss of generality that $u_{i+1}=-u_{i}$ and $v_{i+1}=-v_{i}$. By assumption $R(a, b)$ is not a proper power, therefore only two cases are possible for $R(a, b)$, up to cyclic rearrangement: $R(a, b)=a b a^{-1} b^{-1}$ or $R(a, b)=a b^{-1} a^{-1} b$. In both cases we have

$$
f(x, y, z)=x^{2}+y^{2}+z^{2}-x y z-2-2 \cos \left(\frac{2 \pi}{m}\right)=g(x, y, z)+2-2 \cos \left(\frac{2 \pi}{m}\right) .
$$

Since $2-2 \cos \left(\frac{2 \pi}{m}\right) \neq 0$, it is obvious that $g(x, y, z)$ has no zeros on $V$ in this case.

Thus, we have proved for $m \geqslant 3$ that $\Gamma$ is a non-trivial free product with amalgamation.

Now let $m=2$. Then one can consider the group $\Gamma_{1}=\left\langle a, b \mid R^{4}(a, b)=1\right\rangle$. We proved above that $\operatorname{dim} X^{s}\left(\Gamma_{1}\right) \geqslant 2$. It follows from the proof of Theorem 1 that there exists a representation $\rho: \Gamma_{1} \rightarrow \mathrm{SL}_{2}\left(\mathbb{Q}_{p}\right)$ for some prime $p$ such that $\rho\left(\Gamma_{1}\right)$ is dense in $\mathrm{SL}_{2}\left(\mathbb{Q}_{p}\right)$ in the $p$-adic topology. Hence $\rho\left(\Gamma_{1}\right)$ is a non-trivial free product with amalgamation. Let $G=\overline{\rho\left(\Gamma_{1}\right)}$ be the image of $\rho\left(\Gamma_{1}\right)$ in $\operatorname{PSL}_{2}\left(\mathbb{Q}_{p}\right)$. Then it is easy to see that $G$ is also a non-trivial free product with amalgamation. However, $G$ is an epimorphic image of $\Gamma$, therefore $\Gamma$ is a non-trivial free product with amalgamation, as required.

\section{$\S 3$. Proof of Theorem 2}

(1) Suppose that $\Gamma_{n}=\left\langle a, b \mid a^{n}=b^{k}=R^{2}(a, b)=1\right\rangle$, and let $F_{2}=\langle g, h\rangle$ be the free group with generators $g$ and $h$. We set $x=\tau_{g}, \beta=\tau_{h}=2 \cos (\pi / k)$, and $z=\tau_{g h}$. Consider now the equation

$$
Q_{R(g, h)}(x, \beta, z)=0,
$$

where $Q_{R(g, h)}$ is the Fricke polynomial of the element $R(g, h)$ of $F_{2}$. By Lemma 5 we can write (12) in the following form:

$$
A_{0}(x) z^{s}+\cdots+A_{s}(x)=0
$$

where $A_{0}(x)=\prod_{i=1}^{s} P_{u_{i}-1}(x) P_{v_{i}-1}(\beta)$. Since by assumption there exists $i$ such that $\left|u_{i}\right| \geqslant 2$, it follows that $\operatorname{deg} P_{u_{i}-1}(x) \geqslant 1$. Let $x_{0}=-2 \cos \left(\pi / u_{i}\right)$ be one of the 
zeros of $P_{u_{i}-1}(x)$. Then $x-x_{0}$ divides $A_{0}(x)$, say $A_{0}(x)=\left(x-x_{0}\right) B_{0}(x)$, where $B_{0}(x) \in \mathcal{O}[x]$. We write (13) in the following form:

$$
\left(x-x_{0}\right) B_{0}(x) z^{s}+\cdots+A_{s}(x)=0 .
$$

We assume first that all polynomials $A_{1}(x), \ldots, A_{s}(x)$ are multiples of $x-x_{0}$. Then one can write (14) as follows:

$$
\left(x-x_{0}\right) f(x, z)=0,
$$

where $f(x, z)$ is a polynomial in $x$ and $z$. Let $z_{0}$ be an arbitrary element of $\mathbb{C}$ such that $z_{0} \notin \mathcal{O}$, and let $A$ and $B$ be matrices in $\mathrm{SL}_{2}(\mathbb{C})$ such that $\operatorname{tr} A=x_{0}$, $\operatorname{tr} B=\beta$, and $\operatorname{tr} A B=z_{0}$. By construction, the pair of matrices $(A, B)$ defines a representation of $\Gamma_{n}$ into $\mathrm{PSL}_{2}(\mathbb{C})$. Applying Lemma 6 we see that $\Gamma_{n}$ is a non-trivial free product with amalgamation.

Assume now that not all polynomials $A_{1}(x), \ldots, A_{s}(x)$ are multiples of $x-x_{0}$. For example, assume that $A_{1}(x)$ is not a multiple of $x-x_{0}$, and let $\delta=A_{1}\left(x_{0}\right) \in \mathcal{O}$, $\delta \neq 0$, be the residue of $A_{1}(x)$ modulo $x-x_{0}$. We set $c=N_{\mathbb{Q}(\delta) / \mathbb{Q}}(\delta) \in \mathbb{Z}$ and take $S=\{p \in \mathbb{Z}: p$ divides $c\}$ for the finite set of primes from the assertion of the theorem. Assume that $n=u_{i} p f$ for some integer $f$ and a prime $p$ not in $S$ such that $u_{i} p \nmid u_{j}$ for $j \neq i$. Let $x_{r}=2 \cos \left(r \pi /\left(p u_{i}\right)\right)$, where $r \not \equiv 0$ $(\bmod p)$, and let $K_{r}=\mathbb{Q}\left(\delta, x_{r}-x_{0}\right)$. By Lemma $7(3)$ we can choose $r$ such that $p$ divides $N_{K_{r} / \mathbb{Q}}\left(x_{r}-x_{0}\right)$ and, by construction, $p$ does not divide $c$. Then $N_{K_{r} / \mathbb{Q}}\left(\delta /\left(x_{r}-x_{0}\right)\right) \notin \mathbb{Z}$, therefore $\delta /\left(x_{r}-x_{0}\right) \notin \mathcal{O}$. Thus,

$$
\frac{A_{1}\left(x_{r}\right)}{x_{r}-x_{0}} \notin \mathcal{O} \text {. }
$$

Furthermore, since $p \nmid r$ and $p u_{i} \nmid u_{j}$ for each $j \neq i$, it follows that $B_{0}\left(x_{r}\right) \neq 0$. We now set $x=x_{r}$ and write equation (14) in the following form:

$$
z^{s}+\frac{A_{1}\left(x_{r}\right)}{\left(x_{r}-x_{0}\right) B_{0}\left(x_{r}\right)} z^{s-1}+\cdots+\frac{A_{s}\left(x_{r}\right)}{\left(x_{r}-x_{0}\right) B_{0}\left(x_{r}\right)}=0 .
$$

Clearly, we have $A_{1}\left(x_{r}\right) /\left(\left(x_{r}-x_{0}\right) B_{0}\left(x_{r}\right)\right) \notin \mathcal{O}$ because $B_{0}\left(x_{r}\right) \in \mathcal{O}$. Hence equation (16) has a root $z_{0}$ outside $\mathcal{O}$. Consider now matrices $A$ and $B$ in $\mathrm{SL}_{2}(\mathbb{C})$ such that

$$
\operatorname{tr} A=x_{r}, \quad \operatorname{tr} B=\beta, \quad \operatorname{tr} A B=z_{0} .
$$

By construction, the pair of matrices $(A, B)$ defines a representation of $\Gamma_{n}$ into $\mathrm{PSL}_{2}(\mathbb{C})$. Applying Lemma 6 we see that $\Gamma_{n}$ is a non-trivial free product with amalgamation.

(2) We keep the notation of part (1). Consider the equation

$$
Q_{R(g, h)}(x, \beta, z)=\gamma_{t},
$$

where $\gamma_{t}=2 \cos (t \pi / m), m \nmid t$. By Lemma 5 we can write (17) in the following form:

$$
\left(x-x_{0}\right) B_{0}(x) z^{s}+\cdots+A_{s}(x)-\gamma_{t}=0 .
$$

Let $x_{r}=2 \cos \left(r \pi /\left(p u_{i}\right)\right)$, where $r \not \equiv 0(\bmod p)$. We claim that there exist $t$ and $r$ such that $\left(A_{s}\left(x_{r}\right)-\gamma_{t}\right) /\left(x_{r}-x_{0}\right) \notin \mathcal{O}$. In fact, let us assume the contrary. 
We consider the case $m=3$ first. Then $\gamma_{1}=1$ and $\gamma_{2}=-1$. Since both numbers

$$
\left(A_{s}\left(x_{r}\right)-1\right) /\left(x_{r}-x_{0}\right), \quad\left(A_{s}\left(x_{r}\right)+1\right) /\left(x_{r}-x_{0}\right)
$$

belong to $\mathcal{O}$, their difference $2 /\left(x_{r}-x_{0}\right)$ belongs to $\mathcal{O}$ for each $r \not \equiv 0(\bmod p)$. By assumption $p \neq 2$, therefore we arrive at a contradiction to Lemma $7(4)$.

Now let $m=2^{l}$. Then $\gamma_{2^{l-1}}=0$ and $\gamma_{2^{l-2}}=\sqrt{2}$. Since both quantities

$$
A_{s}\left(x_{r}\right) /\left(x_{r}-x_{0}\right) \text { and }\left(A_{s}\left(x_{r}\right)-\sqrt{2}\right) /\left(x_{r}-x_{0}\right)
$$

belong to $\mathcal{O}$, their difference $\sqrt{2} /\left(x_{r}-x_{0}\right)$ belongs to $\mathcal{O}$, and $2 /\left(x_{r}-x_{0}\right) \in \mathcal{O}$. Again, we obtain a contradiction to Lemma 7(4).

Thus, we choose $t$ and $r$ such that $\left(A_{s}\left(x_{r}\right)-\gamma_{t}\right) /\left(x_{r}-x_{0}\right) \notin \mathcal{O}$. Since $p \nmid r$ and $p u_{i} \nmid u_{j}$ for each $j \neq i$, it follows that $B_{0}\left(x_{r}\right) \neq 0$. We set $x=x_{r}$ and write (18) in the following form:

$$
z^{s}+\cdots+\frac{A_{s}\left(x_{r}\right)-\gamma_{t}}{\left(x_{r}-x_{0}\right) B_{0}\left(x_{r}\right)}=0 .
$$

By construction $\left(A_{s}\left(x_{r}\right)-\gamma_{t}\right) /\left(\left(x_{r}-x_{0}\right) B_{0}\left(x_{r}\right)\right) \notin \mathcal{O}$, therefore (19) has a root $z_{0}$ outside $\mathcal{O}$. Consider now matrices $A$ and $B$ in $\mathrm{SL}_{2}(\mathbb{C})$ such that

$$
\operatorname{tr} A=x_{r}, \quad \operatorname{tr} B=\beta, \quad \operatorname{tr} A B=z_{0} .
$$

By construction the pair of matrices $(A, B)$ defines a representation of $\Gamma_{n}$ into $\mathrm{PSL}_{2}(\mathbb{C})$. Applying Lemma 6 we see that $\Gamma_{n}$ is a non-trivial free product with amalgamation.

(3) Assume that $m>3$ and $m \neq 2^{l}$. Using the notation of part (2) we claim that there exist $t \not \equiv 0(\bmod m)$ and $r \not \equiv 0(\bmod p)$ such that $\left(A_{s}\left(x_{r}\right)-\gamma_{t}\right) /\left(x_{r}-x_{0}\right) \notin \mathcal{O}$. For assume the contrary: suppose that $\left(A_{s}\left(x_{r}\right)-\gamma_{t}\right) /\left(x_{r}-x_{0}\right) \in \mathcal{O}$ for each $t \not \equiv 0$ $(\bmod m)$ and each $r \not \equiv 0(\bmod p)$.

First, we consider the case when $m$ is odd and a multiple of an integer of the form $4 g+1, g \geqslant 1$, that is, $m=(4 g+1) m_{1}$. We consider the quantities $\delta_{t}=\gamma_{2 t m_{1}}=2 \cos (2 t \pi /(4 g+1)), t=1, \ldots, 2 g$. Then $1+\sum_{i=1}^{2 g} \delta_{i}=0$ as it is the sum of all roots of unity of degree $4 g+1$. Note that $-\delta_{t}=\gamma_{(4 g+1-2 t) m_{1}}$. Set $C_{i}=\left(A_{s}\left(x_{r}\right)-(-1)^{i} \delta_{i}\right) /\left(x_{r}-x_{0}\right)$. Then we have

$$
\sum_{i=1}^{2 g}(-1)^{i} C_{i}=-\sum_{i=1}^{2 g} \frac{\delta_{i}}{x_{r}-x_{0}}=\frac{1}{x_{r}-x_{0}} \in \mathcal{O}
$$

whenever $r \not \equiv 0(\bmod p)$, which contradicts Lemma 7(4).

Assume now that $m$ is odd but has no divisors of the form $4 g+1, g \geqslant 1$. Then $m=4 g+3, g \geqslant 1$. We have $1+\sum_{i=1}^{2 g+1} \gamma_{2 i}=0$, for this is the sum of all roots of unity of degree $4 g+3$. We set $C_{0}=\left(A_{s}\left(x_{r}\right)+\gamma_{1}\right) /\left(x_{r}-x_{0}\right)$ and $C_{i}=\left(A_{s}\left(x_{r}\right)-(-1)^{i} \gamma_{2 i}\right) /\left(x_{r}-x_{0}\right)$ for $i=1, \ldots, 2 g+1$. Then

$$
C_{0}+\sum_{i=1}^{2 g+1}(-1)^{i} C_{i}=\frac{\gamma_{1}-1}{x_{r}-x_{0}} \in \mathcal{O}
$$


We claim that $\gamma_{1}-1 \in \mathcal{O}^{*}$. Since $\gamma_{1}$ is a zero of the polynomial $P_{4 g+2}(\lambda), \gamma_{1}-1$ is a zero of $P_{4 g+2}(\lambda+1)$. The constant term of $P_{4 g+2}(\lambda+1)$ is equal to

$$
P_{4 g+2}(1)=P_{4 g+2}\left(2 \cos \frac{\pi}{3}\right)=\frac{\sin ((4 g+3) \pi / 3)}{\sin (\pi / 3)} \in\{-1,1,0\} .
$$

Note that $P_{4 g+2}(1)=0$ if and only if $4 g+3$ is a multiple of 3 , that is, $g$ is a multiple of 3 . Let $g=3 g_{1}$. Then $4 g+3=12 g_{1}+3=3\left(4 g_{1}+1\right)$, that is, $m$ is a multiple of $4 g_{1}+1$, contradicting our assumptions. Hence $P_{4 g+2}(1)= \pm 1$ and $\gamma_{1}-1 \in \mathcal{O}^{*}$. It now follows from $(19)$ that for each $r \not \equiv 0(\bmod p)$ we have $1 /\left(x_{r}-x_{0}\right) \in \mathcal{O}$. We obtain a contradiction to Lemma 7(4) once more.

Finally, we consider the case when $m$ is even, that is, $m=m_{1} 2^{g}$, where $g \geqslant 1$ and $m_{1}$ is odd and greater than 1 . Consider the quantities $\gamma_{i 2^{g}}=2 \cos \left(i \pi / m_{1}\right)$. Now, arguing just in the case of odd $m$ we obtain a contradiction to Lemma $7(4)$.

Thus, we choose $t$ and $r$ such that $\left(A_{s}\left(x_{r}\right)-\gamma_{t}\right) /\left(x_{r}-x_{0}\right) \notin \mathcal{O}$. Then the constant term in equation (19) does not belong to $\mathcal{O}$ and (19) has a root $z_{0}$ outside $\mathcal{O}$. Let $A, B \in \mathrm{SL}_{2}(\mathbb{C})$ be matrices such that

$$
\operatorname{tr} A=x_{r}, \quad \operatorname{tr} B=\beta, \quad \operatorname{tr} A B=z_{0} .
$$

By construction the pair of matrices $(A, B)$ defines a representation of $\Gamma_{n}$ into $\mathrm{PSL}_{2}(\mathbb{C})$. Applying Lemma 6 we complete the proof of Theorem 2.

Remark. In a number of cases one can obtain more precise information on the decomposition of a generalized triangle group $\Gamma$ into a non-trivial free product with amalgamation. For instance, consider the group $\Gamma_{k}=\langle a, b| a^{2}=b^{k}=$ $\left.\left(a b^{2}\right)^{3}=1\right\rangle$. Then it follows from Theorem 2 that $\Gamma_{k}$ is a non-trivial free product with amalgamation if $k=2 k_{1}$, where $1<k_{1} \neq 2^{l}$. However, it is easy to see that also when $k_{1}=2^{l}, l \geqslant 1$, this group $\Gamma_{k}$ is a non-trivial free product with amalgamation. For let $F_{2}=\langle g, h\rangle$ be a free group and let $x=\tau_{g}=0, y=\tau_{h}$, and $z=\tau_{g h}$. Consider the equation

$$
Q_{g h^{2}}(0, y, z)=y z=2 \cos \frac{\pi}{3}=1 .
$$

Let $y=y_{r}=2 \cos \left(r \pi / 2^{l+1}\right)$. Then by Lemma $7(6)$ we obtain $z_{r}=1 / y_{r} \notin \mathcal{O}$ for each odd $r$. Lemma 6 now shows that $\Gamma_{k}$ is a non-trivial free product with amalgamation.

\section{$\S 4$. Proof of Theorem 3}

First, let $R(a, b)=a^{u_{1}} b^{v_{1}} \cdots a^{u_{s}} b^{v_{s}}$ be a word such that $v=\max _{1 \leqslant i \leqslant s}\left|v_{i}\right| \geqslant 2$. Then by Theorem 2 there exists a prime $p$ such that the group $\Gamma_{1}=\langle a, b| a^{n}=$ $\left.b^{p v}=R^{m}(a, b)=1\right\rangle$ is a non-trivial free product with amalgamation. Since $\Gamma_{1}$ is an epimorphic image of $\Gamma, \Gamma$ is also a non-trivial free product with amalgamation.

One can assume therefore without loss of generality that

$$
R(a, b)=a^{u_{1}} b^{v_{1}} \cdots a^{u_{s}} b^{v_{s}},
$$


where $v_{i} \in\{-1,1\}, i=1, \ldots, s$. Assume also that there exists $i, 1 \leqslant i \leqslant s$, such that either $v_{i}=v_{i+1}$ or $v_{1}=v_{s}$. For definiteness, suppose that $v_{1}=v_{2}$. In this case one can consider new generators of $\Gamma$ : $a_{1}=a$ and $b_{1}=a^{u_{2}} b^{v_{1}}$. Then it is easy to verify that $\Gamma=\left\langle a_{1}, b_{1} \mid a_{1}^{n}=R_{1}^{m}\left(a_{1}, b_{1}\right)=1\right\rangle$, where $R_{1}\left(a_{1}, b_{1}\right)=a_{1}^{u_{1}^{\prime}} b_{1}^{v_{1}^{\prime}} \cdots a_{1}^{u_{l}^{\prime}} b_{1}^{v_{l}^{\prime}}, l \geqslant$ $1,0<u_{i}^{\prime}<n$, and $v_{i}^{\prime} \neq 0$ for $i=1, \ldots, l$. Moreover, we have $v^{\prime}=\max _{1 \leqslant i \leqslant l}\left|v_{i}^{\prime}\right| \geqslant 2$. However, we have just proved that $\Gamma$ is a non-trivial free product with amalgamation in this case.

Thus, we can assume without loss of generality that

$$
R(a, b)=a^{u_{1}} b a^{u_{2}} b^{-1} \cdots a^{u_{2 k-1}} b a^{u_{2 k}} b^{-1},
$$

where $k \geqslant 1$ and $0<u_{i}<n$ for $i=1, \ldots, 2 k$. We set $c=b a^{-1} b^{-1}$. Then

$$
R(a, b)=a^{u_{1}} c^{-u_{2}} \cdots a^{u_{2 k-1}} c^{-u_{2 k}}=R_{1}(a, c) .
$$

Let $F_{2}=\langle g, h\rangle$ be the free group of rank 2 and set $f=h g^{-1} h^{-1}$. We set $x=$ $\tau_{g}, y=\tau_{h}, z=\tau_{g h}$, and $t=\tau_{g f}$. Then $\tau_{f}=\tau_{g}=x$ and, by Lemma 9(1), $t=\tau_{g f}=\tau_{g h g^{-1} h^{-1}}=x^{2}+y^{2}+z^{2}-x y z-2$. We regard $R_{1}(g, f) \in F_{2}$ as a word in $g$ and $f$. Let $q(x, t)$ be the Fricke polynomial of $R_{1}(g, f)$, that is,

$$
q(x, t)=Q_{R_{1}(g, f)}\left(\tau_{g}, \tau_{f}, \tau_{g f}\right)=Q_{R_{1}(g, f)}(x, x, t) .
$$

Since $R_{1}(g, f)$ contains $k$ blocks of the form $g^{u_{j}} f^{-u_{j+1}}, q(x, t)$ is a $t$-polynomial of degree $k$ by Lemma 5 , with leading coefficient $(-1)^{k} \prod_{i=1}^{2 k} P_{u_{i}-1}(x)$. By construction $R(g, h)=R_{1}(g, f)$, therefore

$$
Q_{R(g, h)}(x, y, z)=q(x, t)=q\left(x, x^{2}+y^{2}+z^{2}-x y z-2\right) .
$$

We now set $x=\tau_{g}=\alpha_{r}=2 \cos (r \pi / n)$ and $\gamma_{l}=2 \cos (l \pi / m)$, where $r \not \equiv 0(\bmod n)$ and $l \not \equiv 0(\bmod m)$, and consider the equation

$$
Q_{R(g, h)}\left(\alpha_{r}, y, z\right)=\gamma_{l}
$$

By (21) one can write (22) in the following form:

$$
q\left(\alpha_{r}, t\right)=\gamma_{l}
$$

Lemma 10. There exist $r, l$ in $\mathbb{Z}, r \not \equiv 0(\bmod n)$ and $l \not \equiv 0(\bmod m)$, such that $P_{u_{i}-1}\left(\alpha_{r}\right) \neq 0$ for $i=1, \ldots, 2 k$ and equation $(23)$ has a root $t=t_{0} \neq 2$.

Proof. Assume first that $m \geqslant 3$. In this case $\gamma_{1} \neq \gamma_{2}$. We set $r=1$, and then the polynomial $q\left(\alpha_{1}, t\right)$ has degree $k$. Obviously, at least one of the equations $q\left(\alpha_{1}, t\right)=\gamma_{1}$ and $q\left(\alpha_{1}, t\right)=\gamma_{2}$ has a root $t_{0} \neq 2$.

We assume now that $m=2$ and the equation $q\left(\alpha_{r}, t\right)=0$ has the unique root $t=2$. This means that for arbitrary matrices $A$ and $B$ in $\mathrm{SL}_{2}(\mathbb{C})$ such that $\operatorname{tr} A=\operatorname{tr} B=\alpha_{r}$ the condition $\operatorname{tr} R_{1}(A, B)=\operatorname{tr} A^{u_{1}} B^{-u_{2}} \cdots A^{u_{2 k-1}} B^{-u_{2 k}}=0$ yields that $\operatorname{tr} A B=2$. We claim that this is not the case. To obtain a contradiction it is sufficient to find matrices $A$ and $B$ in $\mathrm{SL}_{2}(\mathbb{C})$ satisfying the conditions

(1) $\operatorname{tr} A=\operatorname{tr} B=\alpha_{r}$,

(2) $\operatorname{tr} A B \neq 2$,

(3) $\operatorname{tr} R_{1}(A, B)=\operatorname{tr} A^{u_{1}} B^{-u_{2}} \cdots A^{u_{2 k-1}} B^{-u_{2 k}}=0$. 
We shall seek $A$ and $B$ in the following form:

$$
A=\left(\begin{array}{cc}
\varepsilon_{r} & w \\
0 & \varepsilon_{r}^{-1}
\end{array}\right), \quad B=\left(\begin{array}{cc}
\varepsilon_{r} & 0 \\
w & \varepsilon_{r}^{-1}
\end{array}\right)
$$

where $\varepsilon_{r}+\varepsilon_{r}^{-1}=\alpha_{r}=2 \cos (r \pi / n)$ and $w$ is a variable. It is easy to see that $\operatorname{tr} A B=w^{2}+\varepsilon_{r}^{2}+\varepsilon_{r}^{-2}$. Hence the condition $\operatorname{tr} A B \neq 2$ is equivalent to the inequality $w^{2}+\varepsilon_{r}^{2}+\varepsilon_{r}^{-2} \neq 2$, that is,

$$
w^{2} \neq 2-\left(\varepsilon_{r}^{2}+\varepsilon_{r}^{-2}\right)=2-2 \cos \left(\frac{2 r \pi}{n}\right)=4 \sin ^{2}\left(\frac{r \pi}{n}\right) .
$$

It can be easily verified by induction that

$$
A^{i}=\left(\begin{array}{cc}
\varepsilon_{r}^{i} & P_{i-1}\left(\alpha_{r}\right) w \\
0 & \varepsilon_{r}^{-i}
\end{array}\right), \quad B^{i}=\left(\begin{array}{cc}
\varepsilon_{r}^{i} & 0 \\
P_{i-1}\left(\alpha_{r}\right) w & \varepsilon_{r}^{-i}
\end{array}\right) .
$$

Next, it is not difficult to show that

$$
R_{1}(A, B)=\left(\begin{array}{cc}
\varepsilon_{r}^{d}+C_{1}\left(\alpha_{r}\right) w^{2}+\cdots+C_{k}\left(\alpha_{r}\right) w^{2 k} & w f_{1}(w) \\
w f_{2}(w) & \varepsilon_{r}^{-d}+D_{1}\left(\alpha_{r}\right) w^{2}+\cdots+D_{k-1}\left(\alpha_{r}\right) w^{2 k-2}
\end{array}\right),
$$

where $d=\sum_{i=1}^{2 k} u_{i}, C_{k}\left(\alpha_{r}\right)=(-1)^{k} \prod_{i=1}^{2 k} P_{u_{i}-1}\left(\alpha_{r}\right)$, and $f_{1}(w)$ and $f_{2}(w)$ are some polynomials of $w$. Hence

$$
\operatorname{tr} R_{1}(A, B)=C_{k}\left(\alpha_{r}\right) w^{2 k}+\cdots+\left(C_{1}\left(\alpha_{r}\right)+D_{1}\left(\alpha_{r}\right)\right) w^{2}+\left(\varepsilon_{r}^{d}+\varepsilon_{r}^{-d}\right)=g\left(w^{2}\right) .
$$

We claim that there exists $r, 1 \leqslant r<n$, such that $C_{k}\left(\alpha_{r}\right) \neq 0$ and the polynomial $g\left(w^{2}\right)$ has a root $w_{0}$ such that $w_{0}^{2} \neq 4 \sin ^{2}(r \pi / n)$. Assume the contrary: assume that for each $r$ such that $C_{k}\left(\alpha_{r}\right) \neq 0$ we have

$$
g\left(w^{2}\right)=C_{k}\left(\alpha_{r}\right)\left(w^{2}-4 \sin ^{2}\left(\frac{r \pi}{n}\right)\right)^{k}
$$

Comparing the constant terms in the left-hand and the right-hand sides of (24) and taking the expression for $C_{k}\left(\alpha_{r}\right)$ into account we obtain

$$
\left(\prod_{i=1}^{2 k} P_{u_{i}-1}\left(2 \cos \left(\frac{r \pi}{n}\right)\right)\right) 4^{k}\left(\sin \left(\frac{r \pi}{n}\right)\right)^{2 k}=2 \cos \left(\frac{d r \pi}{n}\right) .
$$

By $(2), P_{u_{i}-1}(2 \cos (r \pi / n))=\sin \left(u_{i} r \pi / n\right) / \sin (r \pi / n)$. We write $u_{i} / n$ as $u_{i}^{\prime} / n_{i}$, where $\left(u_{i}^{\prime}, n_{i}\right)=1$. Then (25) takes the following form:

$$
\prod_{i=1}^{2 k}\left(2 \sin \left(\frac{u_{i}^{\prime} r \pi}{n_{i}}\right)\right)=2 \cos \left(\frac{d r \pi}{n}\right)
$$


Hence to complete the proof of the lemma it is sufficient to show that one obtains a contradiction by assuming that equality (26) holds for each $r$ such that the left-hand side of (26) is distinct from zero.

We start with the discussion of the case of odd $n$. Let $n_{0}=\min _{j} n_{j}$ : assume that $n_{0}=n_{1}$ for definiteness. Then $n_{1}$ is odd; let $p$ be a prime divisor of $n_{1}$. We set $r=n_{1} / p$. Then $2 \sin \left(u_{1}^{\prime} r \pi / n_{1}\right)=2 \sin \left(u_{1}^{\prime} \pi / p\right)$. If $j>1$, then we have $2 \sin \left(u_{j}^{\prime} r \pi / n_{j}\right)=2 \sin \left(u_{j}^{\prime} n_{1} \pi /\left(p n_{j}\right)\right) \neq 0$ because $p n_{j}$ does not divide $u_{j}^{\prime} n_{1}$, by construction. It follows from (26) that

$$
\prod_{i=2}^{2 k}\left(2 \sin \left(\frac{u_{i}^{\prime} n_{1} \pi}{p n_{i}}\right)\right)=\frac{2 \cos \left(d n_{1} \pi /(p n)\right)}{2 \sin \left(u_{1}^{\prime} \pi / p\right)} \in \mathcal{O} .
$$

If $d n_{1}$ is a multiple of $p n$, then $2 \cos \left(d n_{1} \pi /(p n)\right)= \pm 1$. If $d n_{1}$ is not a multiple of $p n$, then $2 \cos \left(d n_{1} \pi /(p n)\right) \in \mathcal{O}^{*}$ by Lemma $7(2)$. In both cases it follows from (27) that $1 /\left(2 \sin \left(u_{1}^{\prime} \pi / p\right)\right) \in \mathcal{O}$, which contradicts Lemma $7(5)$.

Now let $n=2^{l} n^{\prime}$, where $l \geqslant 1$ and $n^{\prime}$ is odd. Let $n_{i}=2^{l_{i}} n_{i}^{\prime}$, where $l_{i} \geqslant 0$ and $n_{i}^{\prime}$ is odd, and let $n_{0}^{\prime}=\min _{j} n_{j}^{\prime}$.

If $n_{0}^{\prime}>1$, then we set $r=2^{l} r^{\prime}$, where $r^{\prime} \not \equiv 0\left(\bmod n^{\prime}\right)$. Now, (26) has the following form:

$$
\prod_{i=1}^{2 k}\left(2 \sin \left(\frac{u_{i}^{\prime} 2^{l-l_{i}} r^{\prime} \pi}{n_{i}^{\prime}}\right)\right)=2 \cos \left(\frac{d r^{\prime} \pi}{n^{\prime}}\right)
$$

where $n^{\prime}$ is odd. We proved above that there exists in this case an $r^{\prime}$ such that the left-hand side of (28) is distinct from zero and equality (28) does not hold.

Now let $n_{0}^{\prime}=1$. We set

$$
I=\left\{i: n_{i}^{\prime}=1\right\}, \quad l_{0}=\min _{i \in I} l_{i}, \quad I_{0}=\left\{i \in I: l_{i}=l_{0}\right\} .
$$

Next we set $r=2^{l_{0}-1} r^{\prime}$, where $r^{\prime}$ is odd. Then for $i$ in $I_{0}$ we have

$$
2 \sin \left(\frac{u_{i}^{\prime} r \pi}{n_{i}}\right)=2 \sin \left(\frac{u_{i}^{\prime} 2^{l_{0}-1} r^{\prime} \pi}{2^{l_{0}}}\right)=2 \sin \left(\frac{u_{i}^{\prime} r^{\prime} \pi}{2}\right)= \pm 2 .
$$

We can now write equality (26) in the following form:

$$
\prod_{i \notin I_{0}}\left(2 \sin \left(\frac{u_{i}^{\prime} r^{\prime} \pi}{2^{l_{i}-l_{0}+1} n_{i}^{\prime}}\right)\right)= \pm \frac{1}{2^{\left|I_{0}\right|-1}} \cos \left(\frac{d r^{\prime} \pi}{2^{l-l_{0}+1} n^{\prime}}\right) .
$$

We choose $r^{\prime}$ such that the left-hand side of (29) is distinct from 0 . Then the right-hand side of (29) is also distinct from 0 . If $\left|I_{0}\right|>1$ or $\left|I_{0}\right|=1$ and $\cos \left(d r^{\prime} \pi /\left(2^{l-l_{0}+1} n^{\prime}\right)\right) \neq \pm 1$, then the left-hand side of (29) belongs to $\mathcal{O}$. However, by Lemma $7(1)$ the right-hand side of (29) does not belong to $\mathcal{O}$, which is a contradiction.

It remains to consider the case $\left|I_{0}\right|=\left|\left\{i_{0}\right\}\right|=1, \cos \left(d r^{\prime} \pi /\left(2^{l-l_{0}+1} n^{\prime}\right)\right)= \pm 1$. In this case (29) has the following form:

$$
\prod_{i \neq i_{0}}\left(2 \sin \left(\frac{u_{i}^{\prime} r^{\prime} \pi}{2^{l_{i}-l_{0}+1} n_{i}^{\prime}}\right)\right)= \pm 1 .
$$


If $|I|>1$ and $i_{0} \neq i \in I$, then $l_{i}>l_{0}$ and $n_{i}=1$. Hence for each odd $r^{\prime}$ the left-hand side of (30) is distinct from 0 and $1 /\left(2 \sin \left(u_{i}^{\prime} r^{\prime} \pi /\left(2^{l_{i}-l_{0}+1}\right)\right)\right) \in \mathcal{O}$ by (30). We arrive at a contradiction to Lemma $7(6)$.

Now let $I=I_{0}=\left\{i_{0}\right\}$. We set

$$
n_{j_{0}}=\min _{j \neq i_{0}} n_{j} \geqslant 3, \quad J=\left\{j: n_{j}=n_{j_{0}}\right\}, \quad l_{j_{0}}=\min _{j \in J} l_{j} .
$$

If $l_{j_{0}}-l_{0}+1>0$, then we set $r^{\prime}=n_{j_{0}}$. It is easy to verify that in this case the left-hand side of (30) is distinct from 0 and it follows from (30) that $1 /\left(2 \sin \left(u_{j_{0}}^{\prime} \pi / 2^{l_{j_{0}}-l_{0}+1}\right)\right) \in \mathcal{O}$. We thus obtain a contradiction to Lemma $7(6)$.

Finally, if $l_{j_{0}}-l_{0}+1 \leqslant 0$, then we consider an arbitrary prime divisor $p, p \geqslant 3$, of $n_{j_{0}}$ and set $r^{\prime}=n_{j_{0}} / p$. Then, as above, the left-hand side of (30) is distinct from zero, and by (30) we obtain

$$
2 \sin \left(\frac{u_{j_{0}}^{\prime} r^{\prime} \pi}{2^{l_{j_{0}}-l_{0}+1} n_{j_{0}}^{\prime}}\right)=2 \sin \left(\frac{u_{j_{0}}^{\prime} 2^{-l_{j_{0}}+l_{0}-1} \pi}{p}\right) \in \mathcal{O}^{*}
$$

This is in contradiction with Lemma 7(5), and it completes the proof of Lemma 10.

We can now complete the proof of Theorem 3. By Lemma 10 we can find $r$ and $l$ such that equation (23) has a root $t_{0} \neq 2$. Since $t=x^{2}+y^{2}+z^{2}-x y z-2$ by construction and $x=\alpha_{r}$, it follows that $y$ and $z$ satisfy the equation

$$
y^{2}+z^{2}-\alpha_{r} y z+\alpha_{r}^{2}-2-t_{0}=0 .
$$

Let $\left(y_{0}, z_{0}\right)$ be a solution of $(31)$ and let $A$ and $B$ be matrices in $\mathrm{SL}_{2}(\mathbb{C})$ such that $\operatorname{tr} A=\alpha_{r}, \operatorname{tr} B=y_{0}, \operatorname{tr} A B=z_{0}$. Then by construction $\operatorname{tr} A B A^{-1} B^{-1}=t_{0}$, $\operatorname{tr} R(A, B)=\gamma_{l}$, and the pair of matrices $(A, B)$ defines a representation of $\Gamma$ into $\mathrm{PSL}_{2}(\mathbb{C})$. Note that this is an irreducible representation because $t_{0} \neq 2$. We claim that there exists a solution $\left(y_{0}, z_{0}\right)$ of equation (31) such that the following conditions hold:

(1) there exists a finite-order element $W_{1}(A, B)=A^{\alpha_{1}} B^{\beta_{1}} \cdots A^{\alpha_{g}} B^{\beta_{g}}$ such that $\alpha_{i}, \beta_{i} \neq 0$ for $i=1, \ldots, g$ and $\sum_{i=1}^{g} \beta_{g} \neq 0$;

(2) $z_{0}=\operatorname{tr} A B \notin \mathcal{O}$.

In that case we can apply Lemma 6 and complete the proof of Theorem 3 . The rest of the roof depends on the form of $t_{0}$. We shall consider the following cases:

(1) $t_{0} \notin \mathcal{O}$;

(2) $t_{0}=2 \cos ((2 k+1) \pi /(2 s+1))$, where $s \geqslant 1$ and $(2 k+1,2 s+1)=1$;

(3) $t_{0}=2 \cos (2 k \pi /(2 s+1))$, where $s \geqslant 1$ and $(k, 2 s+1)=1$;

(4) $t_{0}=2 \cos ((2 k+1) \pi /(2 s))$, where $s \geqslant 1$ and $(2 k+1, s)=1$;

(5) $t_{0} \in \mathcal{O}, t_{0} \neq 2 \cos (k \pi / s)$ for arbitrary integers $k$ and $s$.

(1) We set $y_{0}=0$ and $W_{1}(A, B)=B$, and so $W_{1}(A, B)$ has order 4 . Since $t_{0} \notin \mathcal{O}$, equation $(31)$ has a solution $\left(0, z_{0}\right)$ such that $z_{0} \notin \mathcal{O}$.

(2) We set $W_{1}(A, B)=A B\left(A B A^{-1} B^{-1}\right)^{s}$. Combining Lemmas 8 and 9 we obtain

$$
\operatorname{tr} W_{1}(A, B)=\left(P_{s+1}\left(t_{0}\right)-P_{s}\left(t_{0}\right)\right) z_{0}=0 \cdot z_{0}=0 .
$$


Hence $W_{1}(A, B)$ has order 4 . We now consider an arbitrary solution $\left(y_{0}, z_{0}\right)$ of equation (31) such that $z_{0} \notin \mathcal{O}$.

(3) We set $W_{1}(A, B)=A B\left(A B A^{-1} B^{-1}\right)^{s}$ and assume that

$$
\operatorname{tr} W_{1}(A, B)=2 \cos \frac{\pi}{3}=1 .
$$

Then $W_{1}(A, B)$ has order 6 and it follows from Lemma 9(2) that

$$
\operatorname{tr} W_{1}(A, B)=\left(P_{s+1}\left(t_{0}\right)-P_{s}\left(t_{0}\right)\right) z_{0}=1 .
$$

Hence by Lemma $8(3)$ we obtain $z_{0}=1 /\left(P_{s+1}\left(t_{0}\right)-P_{s}\left(t_{0}\right)\right) \notin \mathcal{O}$. Now let $\left(y_{0}, z_{0}\right)$ be an arbitrary solution of (31). that

(4) We set $W_{1}(A, B)=(A B)^{-1}\left(A B A^{-1} B^{-1}\right)^{s}(A B)^{2}\left(A B A^{-1} B^{-1}\right)^{s}$ and assume

$$
\operatorname{tr} W_{1}(A, B)=2 \cos \frac{\pi}{3}=1 .
$$

Then $W_{1}(A, B)$ has order 6 and by Lemma $9(3)$ we can write (32) in the following form:

$$
\left(t_{0}-2\right) P_{s-1}\left(t_{0}\right)^{2} z_{0}^{3}+\left(2-P_{2 s-1}\left(t_{0}\right)+P_{2 s-2}\left(t_{0}\right)\right) z_{0}-1=0 .
$$

By Lemma $8(4)$ we obtain $0 \neq P_{s-1}\left(t_{0}\right) \notin \mathcal{O}^{*}$, therefore

$$
\frac{1}{\left(t_{0}-2\right) P_{s-1}\left(t_{0}\right)^{2}} \notin \mathcal{O} \text {. }
$$

Thus, (33) has a root $z_{0}$ outside $\mathcal{O}$. Now let $\left(y_{0}, z_{0}\right)$ be a solution of (31).

(5) Since $t_{0} \in \mathcal{O}$ and $t_{0} \neq 2 \cos (k \pi / s)$ for arbitrary integers $k$ and $s$, by Lemma 8(5) there exists an integer $l>0$ such that $0 \neq P_{l}\left(t_{0}\right) \notin \mathcal{O}^{*}$. We set $W_{1}(A, B)=(A B)^{-1}\left(A B A^{-1} B^{-1}\right)^{l+1}(A B)^{2}\left(A B A^{-1} B^{-1}\right)^{l+1}$ and assume that (32) holds. Then $W_{1}(A, B)$ has order 6 and by Lemma $9(3)$ we can write (32) as follows:

$$
\left(t_{0}-2\right) P_{l}\left(t_{0}\right)^{2} z_{0}^{3}+\left(2-P_{2 l+1}\left(t_{0}\right)+P_{2 l}\left(t_{0}\right)\right) z_{0}-1=0 .
$$

Since $1 /\left(\left(t_{0}-2\right) P_{l}\left(t_{0}\right)^{2}\right) \notin \mathcal{O}$ by construction, $(34)$ has a root $z_{0} \notin \mathcal{O}$. Now let $\left(y_{0}, z_{0}\right)$ be a solution of (31). Applying Lemma 6 we complete the proof of Theorem 3 in the last case.

\section{Bibliography}

[1] R. C. Lyndon and P. E. Schupp, Combinatorial group theory, Springer-Verlag, Berlin 1977.

[2] Homological group theory (Durham, 1976), London Math. Soc. Lecture Note Ser., vol. 36, Cambridge Univ. Press, Cambridge 1977.

[3] G. Baumslag and P.B. Shalen, "Amalgamated products and finitely presented groups", Comment. Math. Helv. 65 (1990), 243-254.

[4] B. Fine, F. Levin, and G. Rosenberger, "Free subgroups and decompositions of one-relator products of cyclics. Part 2. Normal torsion free subgroups and FPA decompositions", J. Indian Math. Soc. 49 (1985), 237-247.

[5] H. Zieschang, "On decompositions of discontinuous groups of the plane", Math. Z. 151 (1976), 165-188.

[6] G. Rosenberger, "Bemerkungen zu einer Arbeit von H. Zieschang", Arch. Math. (Basel). 29 (1977), 623-627. 
[7] D. D. Long, C. Maclachlan, and A. W. Reid, "Splitting groups of signature (1,n)", J. Algebra. 185 (1996), 329-341.

[8] M. J. Dunwoody and Sageev M., "Splittings of certain Fuchsian groups", Proc. Amer. Math. Soc. 125:7 (1997), 1953-1954.

[9] V. V. Benyash-Krivets, "Decomposing one-relator products of cyclic groups into free products with amalgamation", Mat. Sb. 189:8 (1998), 13-26; English transl. in Sb. Math. 189 (1998).

[10] V. V. Benyash-Krivets, "On the decomposition of some $F$-groups into free products with amalgamation", Dokl. Akad. Nauk Belarusi 41:6 (1997), 1-4. (Russian)

[11] A. Lubotzky and A. Magid, Varieties of representations of finitely generated groups, Memoirs Amer. Math. Soc., vol. 58, Amer. Math. Soc., Providence, RI 1985.

[12] M. Culler and P. Shalen, "Varieties of group representations and splittings of 3-manifolds", Ann. of Math. 117 (1983), 109-147.

[13] A. S. Rapinchuk, V. V. Benyash-Krivets, and V. I. Chernousov, "Representation varieties of the fundamental groups of compact orientable surfaces", Israel J. Math. 93 (1996), 29-71.

[14] V. V. Benyash-Krivets and V. I. Chernousov, "Representation varieties of the fundamental groups of compact non-orientable surfaces", Mat. Sb. 188:7 (1997), 47-92; English transl. in Sb. Math. 188 (1997).

[15] D. Mumford, Algebraic geometry. I. Complex projective varieties, Imported Publications, Chicago 1979.

[16] R. Horowitz, "Characters of free groups represented in the two dimensional linear group", Comm. Pure. Appl. Math. 25 (1972), 635-649.

[17] H. Helling, "Diskrete Untergruppen von $\mathrm{SL}_{2}(\mathbb{R})$ ", Invent. Math. 17 (1972), 217-229.

[18] W. Magnus, "The uses of 2 by 2 matrices in combinatorial group theory", Results Math. 4:2 (1981), 171-192.

[19] J.-P. Serre, "Arbres, amalgames, $\mathrm{SL}_{2}$ ", Astérisque. 46 (1977).

[20] N. Bourbaki, Groupes et algébres de Lie, Chap. 4, 5 et 6, Masson, Paris 1976.

[21] A. Majeed and A. W. Mason, "Solvable-by-finite subgroups of GL $(2, F)$ ", Glasgow Math. J. 19 (1978), 45-48.

[22] J.-P. Serre, Lie algebras and Lie groups, Springer, Berlin 1992.

[23] È. B. Vinberg, "Rings of definition of dense subgroups of semisimple linear groups", Izv. Akad. Nauk SSSR Ser. Mat. 35:1 (1971), 45-55; English transl. in Math. USSR Izv. 5 (1971).

[24] I. R. Shafarevich, Basic algebraic geometry, Nauka, Moscow 1972; English transl., SpringerVerlag, Berlin 1974.

[25] C. Traina, "Trace polynomial for two-generated subgroups of $\mathrm{SL}_{2}(\mathbb{C})$ ", Proc. Amer. Math. Soc. 79 (1980), 369-372.

[26] H. Bass, "Finitely generated subgroups of $\mathrm{GL}_{2}(\mathbb{C})$ ", The Smith Conjecture, Wiley, New York 1984, pp. 127-136.

Institute of Mathematics,

National Academy of Sciences of Belarus, Minsk

E-mail address: benyash@im.bas-net.by

Received 09/NOV/99

Translated by V. BENYASH-KRIVETS 